\title{
Lipid islands on liquid crystal shells
}

\author{
Anjali Sharma $\odot,{ }^{1,2}$ Deepak Gupta $\odot,{ }^{3,4}$ Giusy Scalia $\odot,{ }^{1}$ and Jan P. F. Lagerwall $\oplus^{1, *}$ \\ ${ }^{1}$ Department of Physics and Materials Science, University of Luxembourg, 162 a Avenue de la faiencerie, 1511 Luxembourg, Luxembourg \\ ${ }^{2}$ Martin A. Fisher School of Physics, Brandeis University, Waltham, Massachusetts 02453, USA \\ ${ }^{3}$ Dipartimento di Fisica 'G. Galilei', INFN, Universitá di Padova, Via Marzolo 8, 35131 Padova, Italy \\ ${ }^{4}$ Department of Physics, Simon Fraser University, Burnaby, British Columbia V5A 1S6, Canada
}

(Received 7 October 2021; accepted 18 January 2022; published 18 February 2022)

\begin{abstract}
By inducing phase separation in lipid monolayers on liquid crystal (LC) shells—thin hollow spheres of LC with water inside and outside-we reveal a rich set of coupled two- and three-dimensional (2D and 3D) selforganization phenomena enabled by the dual closely spaced internal and external spherical LC-water interfaces. Spindle-shaped 2D islands of condensed lipid monolayer first form at the primary interface where lipids are deposited, later also at the initially unexposed secondary interface, because lipids transfer through the LC. The LCs' elastic response to the 3D deformation caused by islands moves them from thin to thick regions on the shell and creates an attraction between opposite-side islands, topologically separated by the LCs, until they stack in a sandwich-like manner. We propose that the phase separation may be used for studying liposome adsorption on soft hydrophobic substrates, and to create unconventional colloidal particles with programmed interactions.
\end{abstract}

DOI: 10.1103/PhysRevResearch.4.013130

\section{INTRODUCTION}

Liquid crystals (LCs) brought into spherical geometry in droplets or shells are powerful model systems for studying the effects of confinement and topological constraints on the fluid long-range orientational order that is the hallmark of LCs $[1,2]$. In a shell, the LC is confined between two aqueous solutions, an outer continuous phase and an internal droplet. This opens attractive perspectives from a soft matter physics point of view, enabling the study of LC ordering in a self-closing phase without edges but with two closely spaced but never overlapping interfaces, with opposite signs of nonzero mean curvature (as defined from within the LC). With the distance between these interfaces on the order of $d \approx 1 \mu \mathrm{m}$, increasing continuously from top to bottom or vice versa due to density mismatch between the LC and the inner droplet, shells have ideal geometry and scale for studying the unique competition between interfacial and bulk aspects of LC self-organization. The former is quantified by the anchoring strength $W$, describing how strongly the director $\mathbf{n}$ (the principle symmetry axis of the nematic phase) is controlled by the boundary conditions. The bulk aspect is accounted for by an effective elastic constant $K$, quantifying the unique nematic elastic response of LCs which counteracts spatial variations of $\mathbf{n}$, i.e., promoting a deformation-free director field $\mathbf{n}(\mathbf{r})$, where $\mathbf{r}$ is the spatial

\footnotetext{
*Corresponding author: jan.lagerwall@1csoftmatter.com

Published by the American Physical Society under the terms of the Creative Commons Attribution 4.0 International license. Further distribution of this work must maintain attribution to the author(s) and the published article's title, journal citation, and DOI.
}

coordinate. A significant anchoring-induced deformation of $\mathbf{n}(\mathbf{r})$ is allowed only if $d>K / W \approx 1 \mu \mathrm{m}$ [3].

Because different configurations of $\mathbf{n}(\mathbf{r})$ yield highly distinct textures in polarizing optical microscopy (POM), due to the LC birefringence, LC shells are also excellent reporters of the presence and behavior of solutes that influence anchoring, yet few solutes have been studied in this regard. The focus has been mainly on the impact of stabilizers added to prevent shell rupture. Water-soluble polymeric stabilizers like polyvinylalcohol (PVA) provide tangential anchoring [4,5], i.e., with n along the interface, while small amphiphilic molecules like surfactants generally promote $\mathbf{n}$ normal to the interface. The potential two-dimensional (2D) organization of adsorbed molecules within an LC shell-water interface was never studied. At flat LC-water interfaces, the Abbott group showed $[6,7]$ that DLPC lipid monolayers can undergo spontaneous 2D phase separation ${ }^{1}$ between condensed and gaslike regions, and that this is clearly visible due to the ability of lipids to impose normal LC anchoring above a threshold coverage $[6,9]$, reached within the condensed regions. Significantly, the phase separation in this case arose as a result of an intricate interplay between the LC-aligning nature of the condensed lipids and the nematic elasticity of the LC [7]; DLPC adsorbed under identical conditions at an isotropic liquid interface is in

\footnotetext{
${ }^{1}$ In line with Gibbs' original definition of coexisting phases as being physically and/or chemically distinct yet having the same chemical potential, we use the term "phase separation" as defined by the International Union of Pure and Applied Chemistry: "The process by which a single solid (liquid) phase separates into two or more new phases" [8]. While this usage is congruent with that of Gupta et al. [7], it may contrast with more specific interpretations of the term in certain communities.
} 
a disordered liquid state and shows no phase separation [7]. As lipids can induce normal anchoring also of LC droplets [10-13], the LC-induced phase separation should take place also on the curved interfaces of LC shells, and we may expect interesting additional phenomena because of the peculiarities of the shell geometry. The LC is now surrounded by aqueous phases on both sides, with opposite signs of curvature on the internal and external interfaces, and phase separated entities are free to move continuously across the entire interface without ever encountering a boundary. Moreover, the shell thickness varies continuously, thereby modulating the elastic forces as function of location in the shell, and consequently also the ability of the LC to promote the lipid phase separation. Yet the response of LC shells to lipids was never explored.

In this paper, we fill this gap, finding a multistage dynamic alignment change in LC shells exposed to DLPC liposome solutions (we denote the aqueous phase in which the liposomes are introduced the primary phase, and the interface of the LC with this phase the primary interface), taking place via $2 \mathrm{D}$ phase separation of lipids adsorbed onto the LCaqueous interfaces of the shells. This allows us to present the first case of LC shells where one and the same interface exhibits regions of orthogonal director alignment coexisting next to each other, as the phase separation results in islands with normal anchoring nucleating within the originally fully tangential-aligned interface. Several previous studies of LC shells demonstrated dynamic changes in alignment, but in contrast to our case, each interface had uniform alignment at any point in time. In contrast to experiments conducted at a single water-LC interface, lipids also transfer through the LC, eventually forming secondary islands at the opposite interface. Although the islands reside on topologically distinct interfaces, with opposite signs of mean curvature, nearby primary and secondary islands experience an attractive interaction via the elastic distortion of $\mathbf{n}(\mathbf{r})$ caused by each island, reminiscent of the attraction between opposite-signed topological defects in LCs.

Beyond soft matter physics insights, our findings show that lipids adsorbing on LC shells have practical value as a versatile platform for designing nontrivial topologies onto responsive materials. We discuss how this platform could be used, e.g., in programming liquid crystal elastomer shells to exhibit sophisticated modes of actuation [14-16] or creating negatively curved particles with van der Waals interactions patterned into complex geometries [17]. It might also find use in biosensing contexts by revealing the presence of unlabeled lipids above a threshold concentration and for improving our understanding of the behavior of lipid monolayers on hydrophobic liquids, biologically relevant through the role of lipid droplets in intracellular cholesterol regulation and lipid metabolism [18].

\section{RESULTS}

\section{A. Primary phase separation on the outside of $\mathrm{LC}$ shells}

Shells are prepared from two common LCs, 5CB (nematic) and 8CB (smectic-A and nematic), with aqueous PVA solutions inside and outside providing tangential anchoring at the start. DLPC is added via a liposome solution, included in the inner phase during shell production, or mixed with the outer phase after the shells have acquired their equilibrium tangential texture. We start by investigating the latter case (see Fig. 1), initially using $8 \mathrm{CB}$ shells that have their thinnest point at the bottom [Figs. 1(a) and 1(b)] because the aqueous inner phase is denser than nematic $8 \mathrm{CB}$. We then study $5 \mathrm{CB}$ shells, for which the density mismatch is inverted, hence they are thinnest at the top.

When a liposome comes into contact with an oil-like liquid such as an LC, it deposits a lipid monolayer at the interface $[19,20]$ as illustrated in Fig. 1(c). Initially, this gives rise to no visible consequence on the LC shells in our experiments [Fig. 1(d)] but after some tens of minutes, polarizing optical microscopy (POM) reveals small spindle-shaped islands with two cusps; see Fig. 1(e). These islands (confirmed also using fluorescence microscopy, see Appendix A and Supplemental Video 1 [21]), which now rapidly grow in size and number during the continued experiment [Figs. 1(f)-1(h)], indicate that $2 \mathrm{D}$ phase separation by nucleation and growth, similar to what was observed by the Abbott group at a flat LC-aqueous interface [7], is indeed taking place also on our LC shells. The reason for the phase separation is a feedback loop between the 2D lipid organization at the LC-aqueous interface and a three-dimensional (3D) elastic deformation of $\mathbf{n}(\mathbf{r})$ caused by the lipid's ability to promote normal LC anchoring if its coverage fraction $\epsilon$ surpasses a threshold level $\epsilon_{c}$. Since the elastic distortion raises Gibbs' free energy density $g$ of the LC, the shell's global Gibbs' free energy $G$ can, when $\epsilon \rightarrow \epsilon_{c}$, be minimized by confining the distortion into small regions with locally increased $\epsilon>\epsilon_{c}$, allowing $\epsilon<\epsilon_{c}$ outside these regions, where the LC thereby avoids the elastic distortion while also respecting the boundary conditions. This is the origin of the initial nucleation of islands, which are thus localized high-energy regions in the shell.

In our study, the phase separation and island formation gives rise to several intriguing phenomena that are unique to LC shells, arising due to three key differences compared to flat samples: (1) a spherical interface has no edges, enabling uninterrupted motion of islands and direct island-island interactions in all directions and at all locations where islands are found, (2) the asymmetric shells naturally have a smooth and tunable thickness gradient that changes the energy cost of island formation and the related elastic deformation as a function of location, and-most significantly-(3) we have not one but two aqueous-LC interfaces, with opposite signs of mean curvature, the secondary being in close vicinity to the primary one. A fourth striking difference is the spontaneous existence of topological defects on LC shells, which might be expected to influence the island formation, but we will see below that their practical impact is insignificant due to the LC's preference to localize defects in the thinnest regions of each shell.

The spindle-like shape of the islands can be recognized also in pictures of phase separation on flat samples, hence it is not unique to shells, but it was not considered in previous reports. The shape is, in fact, quite peculiar, distinctly different from the case of lipid phase separation without the influence of an LC substrate, as in the well-known case of liquid ordered $\left(l_{o}\right)$ rafts separating within mixed liposomes from a surrounding of liquid disordered $\left(l_{d}\right)$ phase [22]. The 

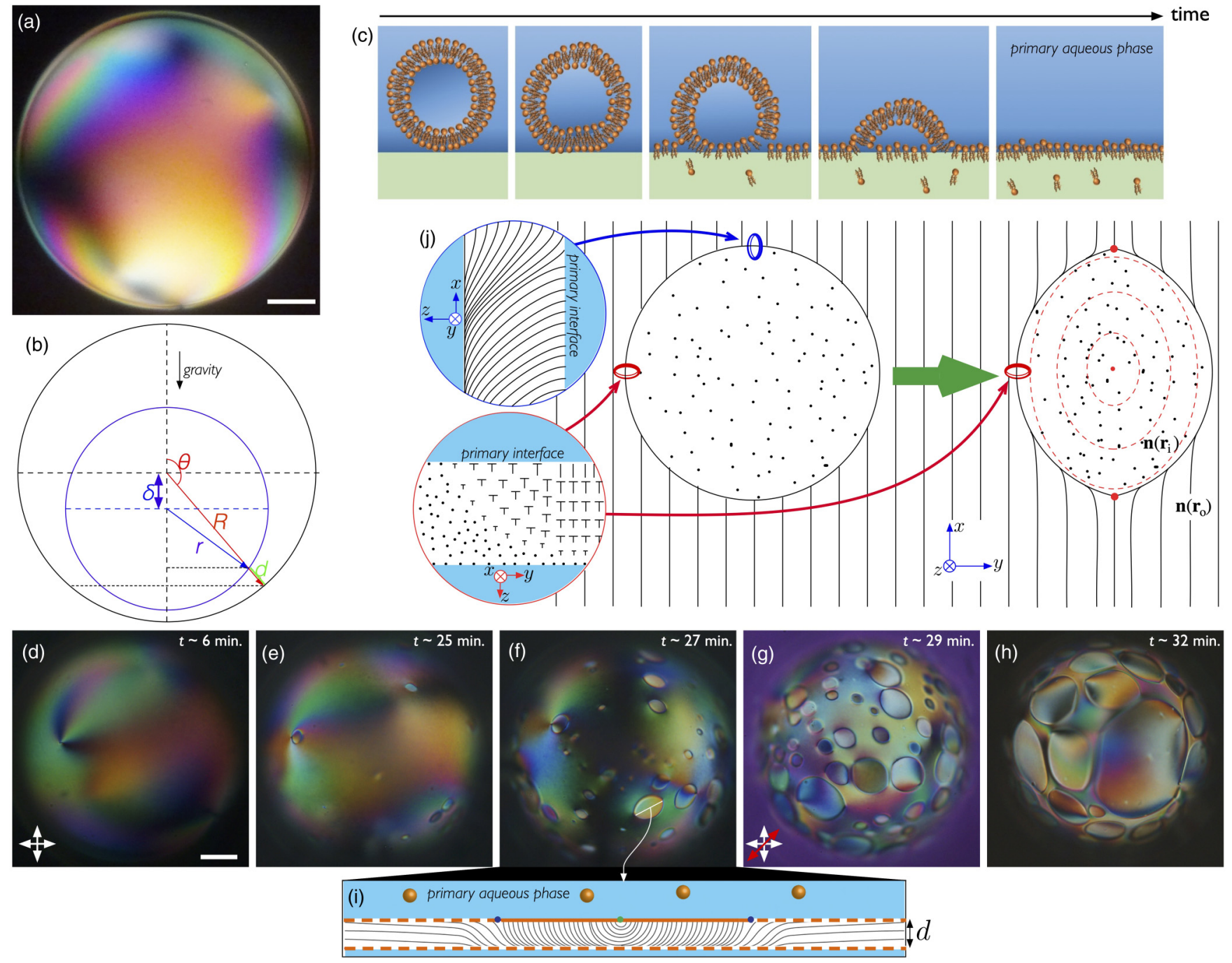

FIG. 1. Island formation on LC shells. (a) Side view of a shell, showing weak asymmetry with thick top and thin bottom. The shell geometry is parametrized in (b). (c) Schematic representation (not to scale) of a liposome (represented by its 2D cross section) depositing on an LC shell, deforming, rupturing, and forming a monolayer at the primary LC-aqueous interface. (d-h) POM images of a nematic shell of $8 \mathrm{CB}\left(35.4{ }^{\circ} \mathrm{C}\right.$, focus on thickest point) with $0.06 \mathrm{mM}$ DLPC in the outer phase (here the primary phase), $t$ the time after adding DLPC. Initially no visible effect is seen (d) but after 25 minutes (e) islands appear and grow (f-h). (i) Schematic showing the director field in a cross section of a shell in the vicinity of an island, as illustrated with respect to (f). The presence of liposomes at the primary interface is indicated with orange spheres (see Fig. 6 for further details). (j) The hydrophobic lipid tails promote normal $\mathbf{n}$ (represented by dots, $\cdot$ ) in the LC in contact with the condensed monolayer in an island while regions outside are tangential (lines, |). The main part shows $\mathbf{n}$ at the primary interface, whereas the insets show cross sections ( $T$ represents $\mathbf{n}$ tilted into the paper plane). The red dashed lines in the right island show the virtual continuation within the island of the tangential director field prevailing outside. It forms a virtual +1 topological defect compensated by two virtual $-1 / 2$ defects at each cusp; islands thus have no net topological charge. Scale bars represent $50 \mu \mathrm{m} ;(\mathrm{g})$ is obtained with a $\lambda$ plate (red double arrow) inserted between the crossed polarizers (white double arrows), giving a clearer view of the external shell boundary.

$l_{o}$ rafts have circular, not spindle-like circumference. The LC substrate thus clearly has an important symmetry-breaking impact, leading to the noncircular island shape in Fig. 1. We therefore begin by analyzing and explaining the island shape and its orientation with respect to the director field in the LC that surrounds an island, $\mathbf{n}\left(\mathbf{r}_{\mathbf{o}}\right)$ ( $o$ for outside the island).

\section{B. Origin of island spindle shape and its orientation in the director field}

Within an island, the LC has switched to normal alignment on the primary side (in contact with the liposome solution) because the condensed 2D lipid phase promotes $\mathbf{n}$ orthogonal to the interface. The other interface (secondary side) remains tangential at this stage, aligned by a PVA solution without liposomes. While this hybrid configuration satisfies the conflicting boundary conditions, it forces the director field inside an island, $\mathbf{n}\left(\mathbf{r}_{\mathbf{i}}\right)$ ( $i$ for inside) to bend by $90^{\circ}$ across the shell thickness $d$, as shown in Fig. 1(i), at an elastic energy cost which increases with decreasing $d$. As stated above, for $d<K / W \approx 1 \mu \mathrm{m}$, elasticity dominates to the point where the anchoring can be violated [3], and we will see below that islands do not form if $d<1 \mu \mathrm{m}$. 
We explain the spindle shape by considering the transition from the hybrid-aligned and deformed $\mathbf{n}\left(\mathbf{r}_{\mathbf{i}}\right)$ inside islands to the tangential director field $\mathbf{n}\left(\mathbf{r}_{\mathbf{o}}\right)$ outside, at different points of the island boundary. When the boundary is perpendicular to $\mathbf{n}\left(\mathbf{r}_{\mathbf{0}}\right)$, as at the top and bottom of the hypothetical circular island on the left in Fig. 1(j), the transition takes place via a bend deformation (blue inset). In contrast, on the left and right the transition is mediated via twist (red inset). The different transitions do not have the same impact on $g$, as becomes clear by analyzing the elastic contribution $g_{e}$ of nematic LCs, following the celebrated Oseen-Frank formalism [23,24]:

$$
\begin{aligned}
g_{e}= & \frac{1}{2} K_{1}[\nabla \cdot \mathbf{n}]^{2}+\frac{1}{2} K_{2}[\mathbf{n} \cdot(\nabla \times \mathbf{n})]^{2} \\
& +\frac{1}{2} K_{3}[\mathbf{n} \times(\nabla \times \mathbf{n})]^{2} .
\end{aligned}
$$

Here $K_{1}, K_{2}$, and $K_{3}$ are the splay, twist, and bend constants, respectively. We omit saddle splay, which has negligible impact on LC shells [25]. Because bend is more costly than twist $\left(K_{3} \approx 5 K_{2}[26,27]\right)$, the twist-mediated boundary is energetically favorable, rendering the line tension anisotropic. It is this symmetry breaking by the LC that deforms islands into spindles, as illustrated on the right in Fig. 1(j). The mediation from $\mathbf{n}\left(\mathbf{r}_{\mathbf{i}}\right)$ to $\mathbf{n}\left(\mathbf{r}_{\mathbf{o}}\right)$ takes place via twist along the entire boundary, the bent mediation relegated to the two cusps. In fact, to maximize the twisted mediation, $\mathbf{n}\left(\mathbf{r}_{\mathbf{o}}\right)$ tends to bend lightly in the plane of the shell near island boundaries, such that the director is everywhere tangential to the island boundary. This can be seen in some of the smaller islands near the shell center in Fig. 1(g), where the boundary is darker along the top left and bottom right, and brighter along the top right and bottom left, compared to the surrounding tangential-aligned LC. We note that the spindle long axis should be along $\mathbf{n}\left(\mathbf{r}_{\mathbf{o}}\right)$ to minimize $g_{e}$. We confirm that this is the case by visualizing $\mathbf{n}\left(\mathbf{r}_{\mathbf{o}}\right)$ by studying the shell in a smectic phase, as demonstrated and explained in Appendix B, Supplemental Video 2 [21], and Fig. 11.

The spindle-shaped islands constitute a $2 \mathrm{D}$ analog of tactoids [29], nuclei of nematic phase coexisting with an isotropic surrounding in lyotropic liquid crystals, adopting a spindle-like shape due to anisotropic interfacial tension [30]. Also tactoids can adopt $2 \mathrm{D}$ character when studied in confinement [31]. A key difference is, however, that tactoids arise as a result of separation between bulk phases of different 3D order, whereas in our case the bulk is uniformly nematic. We instead have a bulk separation into domains with different director configurations $\mathbf{n}\left(\mathbf{r}_{\mathbf{i}}\right)$ and $\mathbf{n}\left(\mathbf{r}_{\mathbf{o}}\right)$. Whereas tactoids acquire their spindle-like 3D shape from the anisotropy in interfacial tension, the spindle-like shape of the condensed lipid islands derive their spindle-like shape from the anisotropy in line tension, and the primary impact is on the $2 \mathrm{D}$ organization of the lipids at the LC-water boundary. Tactoids lose their spindle shape and become more spherical as they grow, in response to the surface-to-volume ratio decreasing, giving less prominence to the interfacial tension anisotropy. The same happens for the islands, which become more circular as opposed to spindle-shaped as they grow, decreasing the perimeter-to-area ratio.

\section{Island stability on different points of a shell}

On shells of low asymmetry, as in Fig. 1, islands are detected everywhere, but less frequently on the thin than on the thick half. Islands born near the thinnest point move to thicker regions with time, whereas islands born on the thick half are stable there. In highly asymmetric shells $[\delta \rightarrow R-r$, where $\delta$ is the vertical offset between the centers of the outer and inner spherical boundaries, with radii $R$ and $r$, respectively; see Fig. 1(b)] this thickness dependence becomes extreme and we cannot detect any islands within a region around the thinnest point, which is the top in the 5CB shells in Fig. 2 and Supplemental Video 3 [21] (see Appendix C for a quantitative analysis). To rationalize this behavior, we extend the thermodynamic model of Gupta et al. [7], derived for a single flat LC-water interface with fixed anchoring on the opposing LC-solid interface, to capture the nontrivial characteristics of our shells with dual curved nonconcentric interfaces, both of which are subject to varying degrees of lipid adsorption, with consequent dynamic anchoring conditions. Because of full rotational symmetry about the vertical axis [polar angle $\theta=0$ or $\pi$; see Fig. 1(b)], Gibbs' free energy $G$ is constant within a conical cross section with the shell, defined by $\theta$ and its variation $\Delta \theta$ (see Fig. 12). We need to consider the unequal interface areas at the insides and outsides, and we get different forms of $G$ for the outside/inside being primary/secondary side, and vice versa. To avoid dependence on the arbitrarily chosen variation $\Delta \theta$, we eliminate $\Delta \theta$ by a simple rescaling $G \rightarrow G^{\prime}$, giving us the expression for outer primary side (see derivation in Appendix D, where also the corresponding result for inner primary side is presented):

$$
\begin{aligned}
G^{\prime}= & \frac{k T}{A^{*}}\left[R^{2} \gamma\left(\epsilon_{p}\right)+(R-d)^{2} \gamma\left(\epsilon_{s}\right)\right] \\
& +\frac{K\left(\alpha_{p}-\alpha_{s}\right)^{2}}{6 d}\left(3 R^{2}-3 d R+d^{2}\right) \\
& +\frac{W}{2}\left[(R-d)^{2} \sin ^{2} \Delta \alpha_{s}+R^{2} \sin ^{2} \Delta \alpha_{p}\right] .
\end{aligned}
$$

The lipid coverage fractions at each LC-aqueous interface are given by $\epsilon_{i}$, with $\epsilon_{i}=1$ corresponding to saturation. The index $i$ indicates whether we consider the primary $(i=p)$ or secondary $(i=s)$ interface. The interfacial mixing contribution to $G^{\prime}$ is captured in classical manner with $\gamma(\epsilon)=\epsilon \ln \epsilon+$ $(1-\epsilon) \ln (1-\epsilon)+\chi \epsilon(1-\epsilon)$, with $\chi$ the Flory interaction parameter, here describing the attractive interaction between lipids at each interface. Gupta et al. found $\chi=2$ to be appropriate for DLPC at a flat LC-water interface at room temperature [7], and we adopt this value as a first approximation. As usual, $W$ and $K$ represent anchoring strength and elastic response of the bulk LC, respectively, the latter appropriate for splay-bend deformation, and $k$ and $T$ are the Boltzmann constant and the absolute temperature, respectively. $A^{*}$ is the area per lipid molecule at maximum coverage, i.e., $\epsilon_{i}=1$. We here approximate its numerical value as $A^{*} \approx 0.4 \mathrm{~nm}^{2}$ for either interface, primary or secondary. This value is taken from the point of collapse of a DLPC monolayer at a flat air-water interface subject to compression [32].

The local shell thickness $d$ is a function of $\theta$ (see Appendix E), and $\alpha_{i}$ are the actual angles that $\mathbf{n}(\mathbf{r})$ makes 


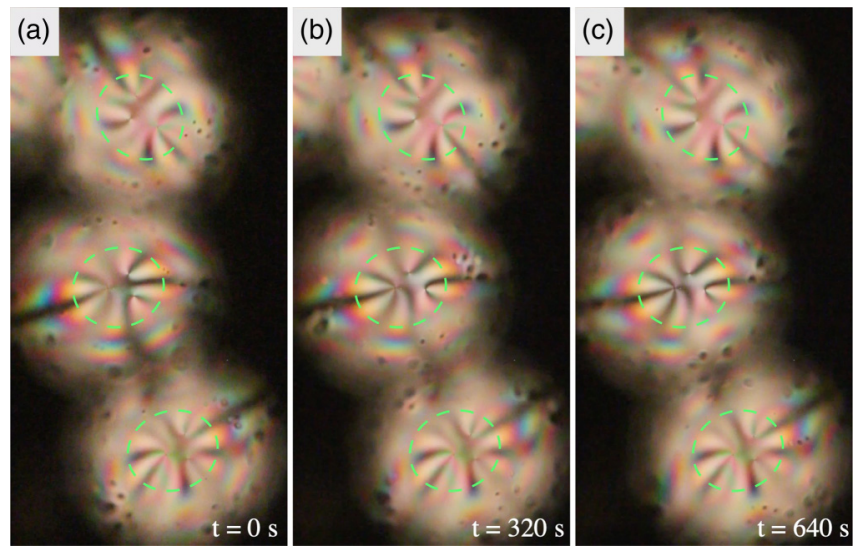

FIG. 2. Island-free regions in highly asymmetric shells. Three consecutive frames from Supplemental Video 3 [21] (quantitative analysis in Appendix C), showing rapid island formation in three highly asymmetric shells (confirmed by all topological defects being near the thinnest point, here the top) of 5CB for $\epsilon_{p}>\epsilon_{c}$. The ellipses indicate the boundaries of the regions near the top of each shell in which the thickness is too low to allow island formation. The distortion of the shells into elliptical rather than circular cross section is an optical artifact explained in [28]. This artifact makes the use of scale bars problematic, but as a reference, the shell radius is about $103 \mu \mathrm{m}$.

to the interface normal at the primary and secondary interfaces, respectively, the deviations from the preferred $\mathbf{n}(\mathbf{r})$ given by $\Delta \alpha_{i}$. As is clear from the derivation in Appendix D [Eq. (D14)], $\Delta \alpha_{i}$ depends sensitively on $\epsilon_{c}$, which is the critical lipid coverage needed to turn the anchoring from promoting tangential to promoting normal director at an interface. Also for $\epsilon_{c}$ we adopt the value reported in [7], $\epsilon_{c} \approx 0.8$. The reason that we use values for $\chi, A^{*}$, and $\epsilon_{c}$ obtained for flat interfaces, although our interfaces are curved, is that they are not trivial to measure experimentally. In Sec. III we will come back to the impact of positive and negative mean curvature, respectively, and how it may influence our quantitative results.

Equation (2) is the fundamental tool necessary to explain the motion of islands as well as their absence at the thinnest regions of highly asymmetric shells, but the situation is further complicated by the fact that we need to compare different subsections of the shell that can have very different partial volumes and interfacial areas, as well as varying values of $d, \epsilon_{p}$ and $\epsilon_{s}$. We cannot simply consider energy densities, because a density is inherently either $2 \mathrm{D}$ or $3 \mathrm{D}$, while in our case interfacial and bulk contributions to $G$ (or $G^{\prime}$ ) compete with similar magnitude. We therefore construct an intensive property $\Delta_{i}$ as the relative change in $G^{\prime}$ resulting from an orthogonal realignment at interface $i$ while the opposing interface remains unchanged:

$$
\Delta_{i}=\frac{G^{\prime}\left(\alpha_{i}=0^{\circ}\right)-G^{\prime}\left(\alpha_{i}=90^{\circ}\right)}{\left|G^{\prime}\left(\alpha_{i}=90^{\circ}\right)\right|} .
$$

The benefit of defining (3) is that we can evaluate it as a function of polar angle $\theta$, the sign of $\Delta_{i}$ telling us whether we should expect island formation or not. Here we are interested in whether a birth of an island on the primary interface of the pristine shell is favorable or not, hence we consider $i=p$ and $\alpha_{s}=90^{\circ}$. A positive $\Delta_{p}$ means that the realignment to normal primary interface raises the free energy, hence no islands should be seen at the selected value of $\theta$, whereas $\Delta_{p}<0$ indicates that island formation is favored, as it reduces $G^{\prime}$.

We plot $\Delta_{p}$ for several values of $\theta$ in Fig. 3, for parameter values corresponding to [Figs. 3(a) and 3(b)] a weakly asymmetric shell with thin bottom (modeled after the shell in Fig. 1) and [Figs. 3(c) and 3(d)] a highly asymmetric shell with thin top (modeled after the shells in Fig. 2). For every curve in Fig. 3(b), we can define a stability limit $\epsilon_{0} \gtrsim \epsilon_{c}$ such that $\Delta_{p}<0$ for primary lipid coverage $\epsilon_{p}>\epsilon_{0}$. This means that the original tangential configuration becomes unstable against hybrid configuration, as within islands, for lipid coverage on the primary interface beyond $\epsilon_{0}$. Note that, while $\epsilon_{0}$ and $\epsilon_{c}$ are closely related and even similar in value here, their meanings are distinct, as $\epsilon_{0}$ considers the impact of interface anchoring and bulk elasticity, while $\epsilon_{c}$ is defined only with respect to the former. For this reason, it is not enough to compare $\epsilon_{p}$ and $\epsilon_{c}$ to judge whether primary islands form or not.

Figure 3(b) also explains the time sequence of events from Fig. 1(d) to Fig. 1(h). During the early stage we see no islands, since the pristine shell starts out without lipids. The initial lipid coverage is thus $\epsilon_{p}=0$, which by definition means that $\Delta_{p}>0$ in the beginning. As lipids adsorb, $\epsilon_{p}$ gradually increases and will eventually reach the stability limit, $\epsilon_{p}=\epsilon_{0}$. From the observation that phase separation sets in and the first hybrid-aligning islands appear after around $25 \mathrm{~min}$ in Fig. 1(e), we can thus infer indirectly that at this point $\epsilon_{p}=\epsilon_{0}$, although we have no means of measuring $\epsilon_{p}$ directly. From this point on, we see in Figs. 1(f)-1(h) how further lipid adsorption grows existing islands and seeds new ones.

The situation is different for the highly asymmetric, thintopped shell modeled in Fig. 3(d). Here we cannot define any stability limit $\epsilon_{0}$ for $\theta<25.1^{\circ}$. At this polar angle, corresponding to $d=1.2 \mu \mathrm{m}$, our model produces $\Delta_{p}=0$ for a primary lipid coverage $\epsilon_{p}=0.86$, but $\Delta_{p}$ never reaches negative values. For smaller $\theta$ yet, $\Delta_{p}>0$ for all $\epsilon_{p}$, hence island formation is unfavored. In general, the $2 \mathrm{D}$ phase separation giving rise to the primary condensed lipid island formation on the shells can be described by a gap in an $\left(\epsilon_{p}, \theta\right)$ phase diagram. On this shell, the gap ends at the critical point $\theta=$ $25.1^{\circ}, \epsilon_{p}=0.86$; see Fig. 4 (see derivation in Appendix F). This is the reason why islands never form within the thinnest region, bounded by $\theta \approx 25^{\circ}$, of the highly asymmetric shells in Fig. 2. We can conclude that Eq. (2) helps understand the experimental observations described above, including quantitative data, on strongly and on weakly asymmetric shells.

\section{Island growth and formation of secondary islands and island sandwiches}

Once island formation on the primary side has started, we are in the coexistence regime between gaslike and condensed $2 \mathrm{D}$ organization in the lipid monolayer at the primary interface. This means that the primary lipid coverage fraction $\epsilon_{p}$ outside islands is saturated at the stability limit $\epsilon_{0}$ (in regions thick enough for $\epsilon_{0}$ to be defined). The fate of further added lipids must then follow one of three scenarios: (1) nucleate a new island, (2) grow an existing island, or (3) transfer to the secondary side of the shell, where an LC-aqueous interface 
(a)

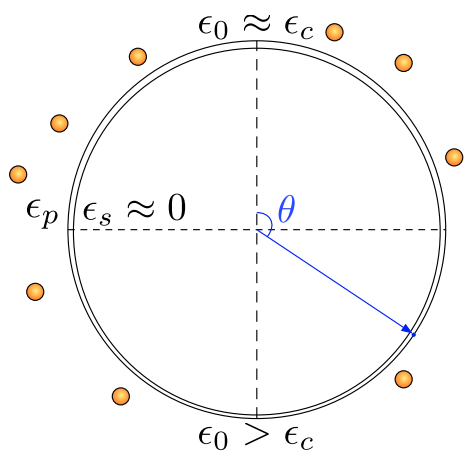

(c)

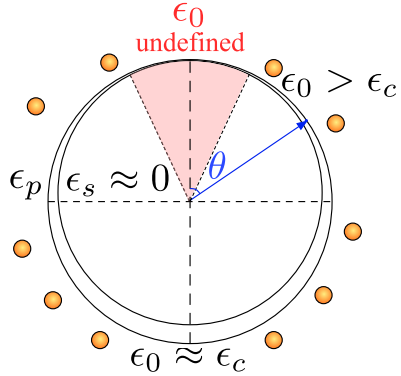

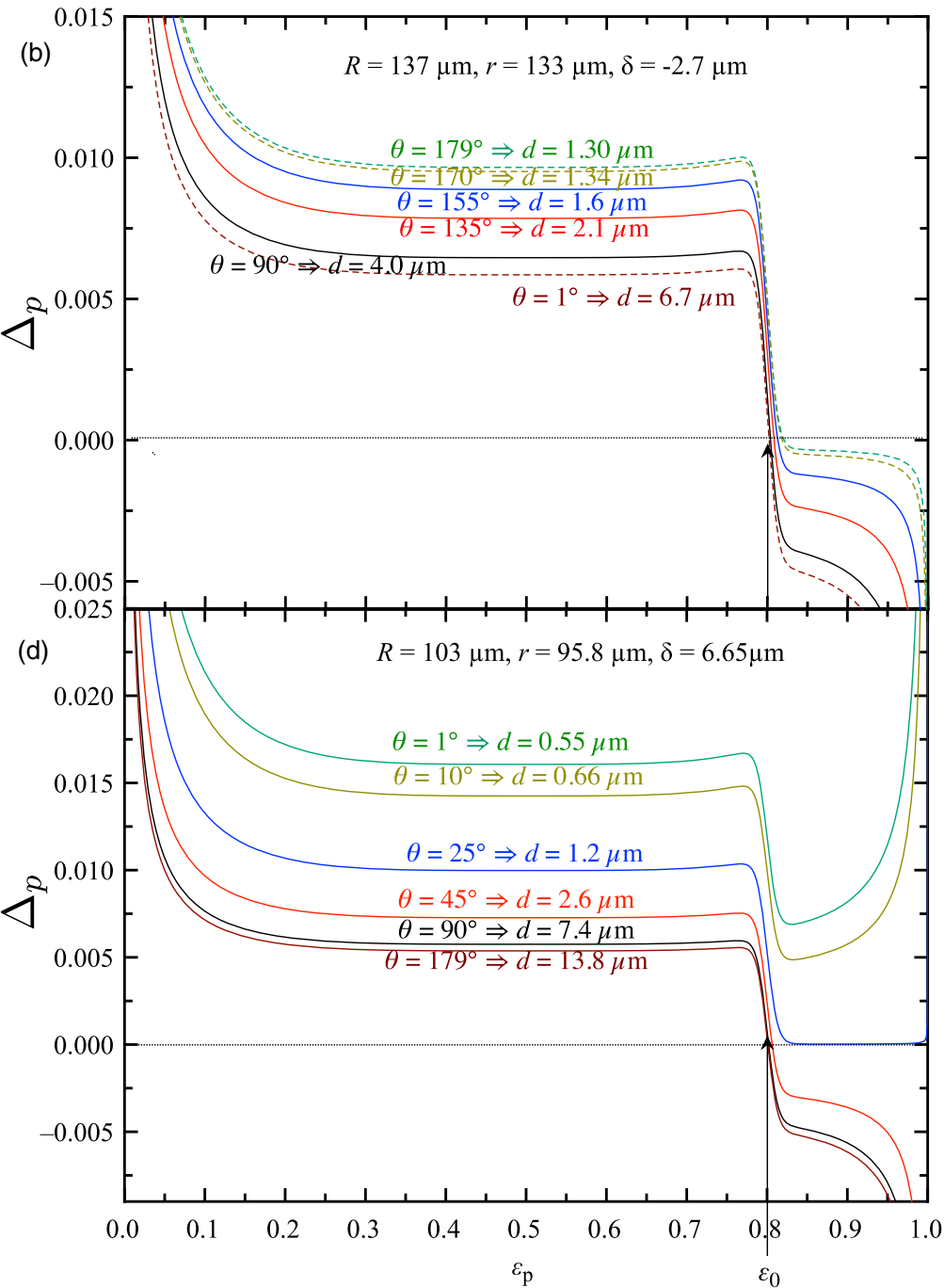

FIG. 3. Relative free energy difference $\Delta_{p}$ between hybrid and tangential director fields as function of lipid packing fraction $\epsilon_{p}$ and location on a shell. The curves are calculated for $(a, b)$ the weakly asymmetric shell with thin bottom in Fig. 1 and (c, d) the highly asymmetric shell with thin top in Fig. 2, different locations on each shell identified by varying the polar angle $\theta$. As illustrated with the external orange spheres in the cartoons in ( $\mathrm{a}$ and $\mathrm{c}$ ), we consider lipid added to the outer phase and the stage prior to any significant lipid transfer through the LC, i.e., the secondary side has negligible lipid coverage, $\epsilon_{s} \approx 0$, and its alignment is tangential, $\alpha_{s}=90^{\circ}$. The bulk elastic deformation if the primary side turns radial is strong on the thin side, increasing the actual lipid coverage threshold $\epsilon_{0}$ for alignment switching with respect to the threshold $\epsilon_{c}$, which neglects elastic energy contributions. The corresponding deformation on the thick side is weak, rendering $\epsilon_{0} \approx \epsilon_{c}$. While island formation is favored at $\epsilon_{p}>\epsilon_{0}$ for any $\theta$ in (a) and (b), this is not the case in (c) and (d), where $\theta<25^{\circ}$ leaves $\epsilon_{0}$ undefined and the initial configuration energy minimizing at all $\epsilon_{p}$. Common parameter values used for all calculations: $\epsilon_{s}=0.0001, \alpha_{s}=90^{\circ}, \chi=2, A^{*}=0.4$ $\mathrm{nm}^{2}, K=10^{-11} \mathrm{~N}, W=20 \mu \mathrm{J} / \mathrm{m}^{2}, B=100, \epsilon_{c}=0.8, T=300 \mathrm{~K}$.

far from saturation is still available. Experimentally, we see all three scenarios, although the third initially gives no visible effect since at the start, the lipid coverage on the secondary interface is $\epsilon_{s}=0 \ll \epsilon_{0}$. We argue that scenarios (1) and (2) are the most likely when a liposome deposits at a point with large $d$, where the elastic energy cost of islands is low while the distance to the secondary interface is long. In contrast, for low $d$ the distortion of $\mathbf{n}(\mathbf{r})$ caused by islands is energetically costly whereas lipid transfer via scenario (3) is likely.

As the number and size of primary islands increase, they eventually come so close that they merge into larger islands [Figs. 5(a)-5(c) and Supplemental Video 4 [21]], minimizing the impact of line tension. Moreover, provided sufficient liposome concentration, scenario (3) will eventually lead to $\epsilon_{s}=\epsilon_{0}$, i.e., also the secondary interface has a lipid coverage that is saturated for 2D gaslike organization. Due to their opposite signs of mean curvature, we expect the insides and outsides to have slightly different values of the stability limit $\epsilon_{0}$, a matter we ignore for now but will come back to in Sec. III. Further lipid transfer will thus lead to phase separation and nucleation of secondary islands with condensed lipid organization on that interface as well. Once secondary islands have formed we see a distinctive type of dynamics if the two types of islands approach; see Figs. 5(d)-5(f) and Supplemental Video 5 [21]. While adjacent same-side islands exhibit slight repulsion between the boundaries prior to merging, islands on opposite shell sides attract if they get close enough (see Appendix G and Fig. 15 for a quantitative analysis of the 


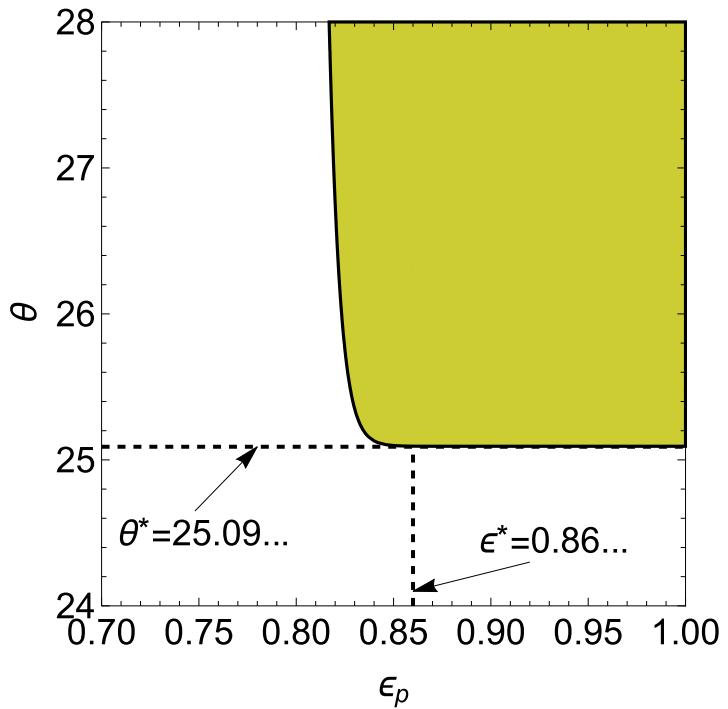

FIG. 4. Critical point in island formation phase diagram. Calculated phase diagram (details in Appendix F) for the primary island formation on a highly asymmetric shell as in Fig. 2, zooming in on the boundary where the shell gets too thin for phase separation to occur. The polar angle $\theta^{*} \approx 25.1^{\circ}$ and lipid coverage $\epsilon^{*} \approx 0.86$ [numerical values correspond to the parameters used in Fig. 3(d)] define a critical point of the phase diagram. In the white region, no islands can form, hence it retains the original tangential configuration.

interaction energies). Significantly, the island borders do not open and fuse as when same-side islands merge, but they both remain intact while the islands superpose and align one on top of the other as a sandwich [Figs. 5(e) and 5(f)]. The LC in between, directed by condensed lipid monolayers at both interfaces, is now normal-aligned without elastic distortion, reducing $G$. Figure 6 illustrates the process of island overlap schematically, considering also the evolution of topological defects within the LC shell cross section. They always add up to zero, as they must, given the flat topology of the cross section.

When two island sandwiches approach, also these merge; see Figs. 5(g)-5(i). The dynamics is different again, as this involves four islands merging pairwise into two. First, the two primary islands merge, the secondary islands remaining distinct on the other side [Fig. 5(h)]. Immediately afterwards, however, also the secondary islands merge, leaving a single normal-aligned sandwich island of large cross section [Fig. 5(i)].

\section{E. The case of inner primary phase}

Given a certain initial lipid concentration $C_{0}$ (in $\mathrm{mM}$ ) and volume $V$ (in $\mathrm{L}$ ) of the aqueous phase to which liposomes are added, we can formulate the maximum possible primary lipid coverage fraction $\epsilon_{p}^{m}$ that could be achieved with the added liposomes - corresponding to a situation where no lipid escapes to the secondary side-by comparing the number of lipid molecules in solution with the available binding sites on the primary interface. The number of primary lipid molecules is $N_{0}=V C_{0} N_{A}$, where $N_{A}$ is Avogadro's constant. By introducing DLPC in the inner phase during shell production we
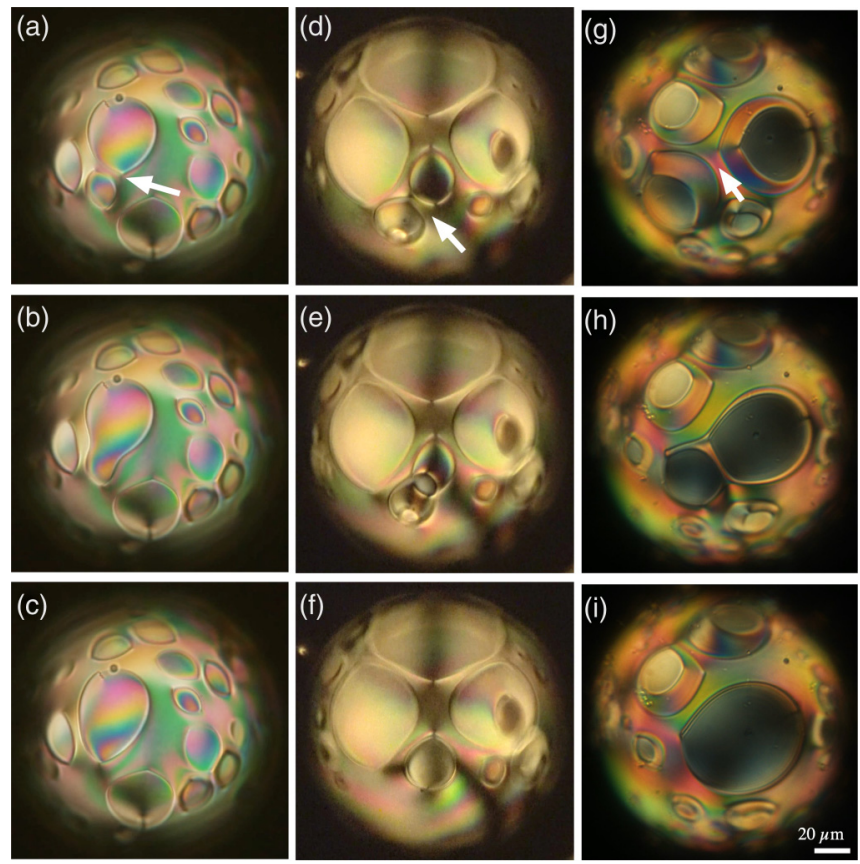

FIG. 5. Island merging/superposition. The top row shows shells with two islands about to merge/superpose (white arrows), the event happening in the middle row. In the bottom row the resulting configuration has relaxed somewhat. (a-c) (from Supplemental Video 4 [21]) Primary (same-side) islands merging in a nematic $8 \mathrm{CB}$ shell; $0.06 \mathrm{mM}$ lipid added to the outer phase. (d-f) (from Supplemental Video 5 [21]) Primary and secondary islands (on opposing interfaces) attracting and superposing as a sandwich in a nematic 8CB shell; $0.06 \mathrm{mM}$ lipid added to the outer phase. The island sandwich appears bright due to birefringence from the back of the shell. The exposure has been increased digitally. (g-i) Island sandwiches merging in a nematic $5 \mathrm{CB}$ shell at room temperature; $0.25 \mathrm{mM}$ lipid was added to the outer phase.

obtain precise control of the primary LC area as well as the number of lipids in the liposome solution with which it is in contact. The fraction between $N_{0}$ and the number of lipids that would saturate the primary inside of a single shell, with area $A_{\mathrm{LC}}^{\text {in }}$ and inner volume $V^{\text {in }}$, can then be written

$$
\epsilon_{p}^{m}=\frac{V^{\text {in }} C_{0} N_{A}}{A_{\mathrm{LC}}^{\text {in }} / A^{*}}=\frac{\frac{4}{3} \pi r^{3} C_{0} N_{A} A^{*}}{4 \pi r^{2}}=\frac{N_{A} A^{*}}{3} C_{0} r .
$$

The inside $\epsilon_{p}^{m}$ thus scales linearly with $A^{*}, C_{0}$, and $r$. The corresponding expression for primary outer phase is more complex, as the total volume of outer phase, $V^{\text {out }}$, can be varied independently of the shell characteristics, and as the corresponding adsorption area $A_{\mathrm{LC}}^{\text {out }}$ scales with the number of shells.

In our experiments with inner primary phase (Appendix $\mathrm{H}$ and Fig. 16), we find that the minimum $C_{0}$ that triggers visible island formation on the inside of shells with $r \approx 88 \mu \mathrm{m}$, is $C_{0} \approx 0.05 \mathrm{mM}$. Using the usual $A^{*}=0.4 \mathrm{~nm}^{2}$, Eq. (4) then gives us an upper estimate of the minimum lipid coverage fraction for island formation on the inside of only $\epsilon_{0}^{\prime} \approx 0.35$. It is worth nothing that the time to detect the first island at this low lipid concentration is much longer than during the experiments described above, about $2 \mathrm{hr}$, and the continued 


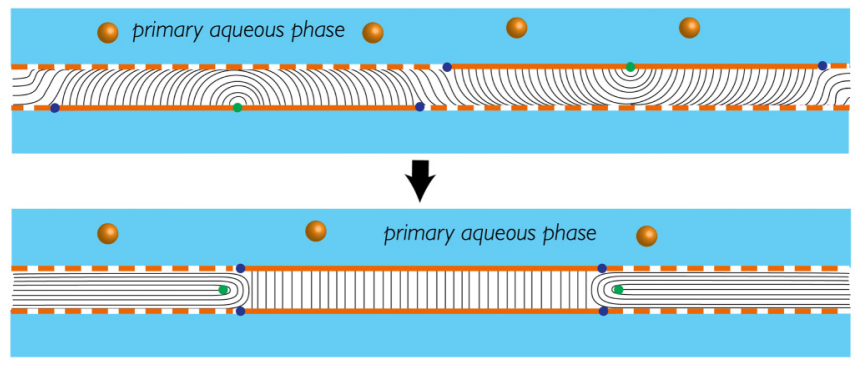

FIG. 6. Primary and secondary islands superposing into a sandwich. In each panel (top: adjacent islands; bottom: superposed island sandwich), the top is the primary side, the orange spheres representing liposomes. Condensed lipid regions at the interfaces are indicated with fully drawn orange boundaries, whereas gaslike lipid monolayers are indicated with dashed orange boundaries. The black lines represent $\mathbf{n}(\mathbf{r})$ in the LC and positive topological defects are drawn in green, negative in blue. Note that defects at an interface to the aqueous phase contribute only half the charge of what a bulk defect with the same type of surrounding director field deformation would yield.

island formation is slower. These observations suggest that phase separation has not set in globally. In Sec. III we will come back to the interesting question of why the calculated $\epsilon_{0}^{\prime}$ for primary phase on the inside appears so much lower than the value of $\epsilon_{0}$ estimated for primary phase on the outside.

\section{F. The dynamics of island formation and disappearance}

To model the dynamics of the formation of primary islands, we assume that the probability of liposome deposition is constant in time, at least up to the point of the first island birth, before any LC-aqueous interface is saturated with lipids. The same should hold for the probability of deposited lipid to transfer from the primary to the secondary side, but here also the reverse process is possible since lipids can transfer through the shell in both directions. We should thus be able to describe the dynamics as a sequence of two Poisson processes, the first irreversible, the second reversible. As detailed in Appendix I, we can formulate an ideal function for the primary lipid coverage $\epsilon_{p}$ as a function of time $t$ by introducing $\lambda_{L p}$ as the rate constant describing the probability of liposomes (index $L$ ) depositing on shells, and $\lambda_{p s}$ the corresponding constant for transfer across the LC (in a first approximation assumed to be identical for both directions of transfer):

$$
\begin{aligned}
\epsilon_{p}(t)= & \frac{A^{*}}{A_{\mathrm{LC}}} \frac{C_{0} V N_{A} e^{-\lambda_{L p} t}}{2\left(\lambda_{L p}-2 \lambda_{p s}\right)}\left[\lambda_{L p}\left(e^{\left(\lambda_{L p}-2 \lambda_{p s}\right) t}+e^{\lambda_{L p} t}-2\right)\right. \\
& \left.-2 \lambda_{p s}\left(e^{\lambda_{L p} t}-1\right)\right] .
\end{aligned}
$$

Since we cannot easily access $\epsilon_{p}(t)$ experimentally, we instead determine the time $t_{0}$ to the first island detection, thus $t_{0}$ corresponds to $\epsilon_{p}=\epsilon_{0}$, at least locally. We can do this experiment only as a function of $C_{0}$ when the external phase is the primary phase, since experiments with internal primary phase require the lipids to be introduced at the point of shell production and we cannot ensure the required constant geometrical parameters for all shells between multiple batches, with varying $C_{0}$. Moreover, because the time $t$ after lipid addition is identical to the lifetime of shells with internal

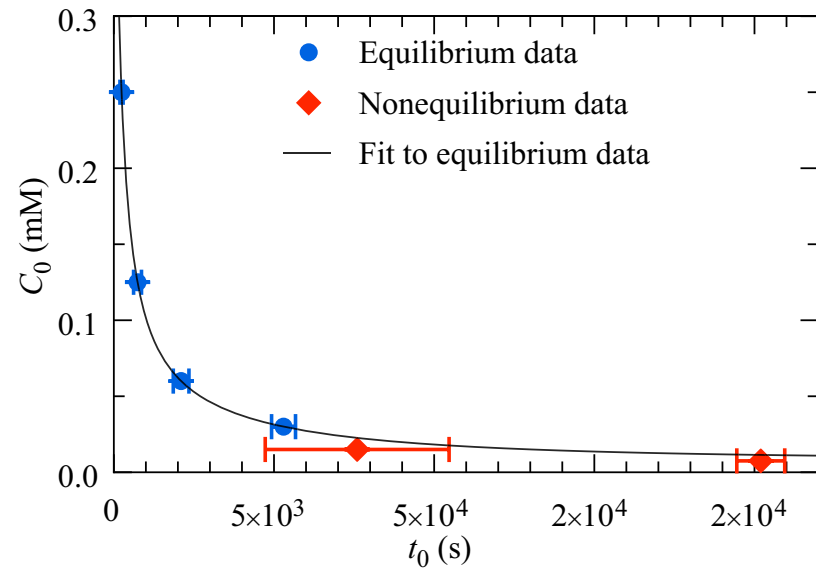

FIG. 7. Lipid concentration vs. island formation time. The primary phase lipid concentration $C_{0}$ is plotted versus $t_{0}$, the time to form the first primary island. Blue dots: equilibrium island formation at global $\epsilon_{p} \geqslant \epsilon_{0}$. Red dots: formation of islands that may be kinetically arrested, with local $\epsilon_{p}=\epsilon_{0}$ but possibly global $\epsilon_{p}<$ $\epsilon_{0}$. The black curve shows a manually assisted fit of Eq. (6) to the equilibrium data points, using small offsets $\Delta t$ and $\Delta C$ in time and concentration, respectively, as motivated in Appendix I. Parameter values of the plotted function: $A^{*}=0.4 \mathrm{~nm}^{2}, A_{\mathrm{LC}}=$ $13.6 \mathrm{~mm}^{2}, V=4.9 \mathrm{~mm}^{3}, \epsilon_{0}=0.8, \lambda_{L p}=10^{-4} \mathrm{~s}^{-1}, \lambda_{p s}=6 \cdot 10^{-4}$ $\mathrm{s}^{-1}, \Delta t=-179 \mathrm{~s}, \Delta C=-10 \mu \mathrm{M}$.

primary phase, it is more difficult to accurately and continuously monitor the shell texture as a function of $t$ for internal primary phase. We thus produce a single large batch of shells without lipids which is then subdivided into several smaller samples with the same number density of shells, and we add lipids to the outer phase, at multiple initial concentrations $C_{0}$, from 7-250 $\mu \mathrm{M}$; see Appendix J and Fig. 18. At $C_{0} \geqslant 60$ $\mu \mathrm{M}$, the entire shell eventually ends up normal-aligned while $C_{0} \leqslant 30 \mu \mathrm{M}$ does not reach such an end state. Thus, at $C_{0}=60 \mu \mathrm{M}, V C_{0}$ provides enough DLPC to condense the lipids across both interfaces, i.e., to ensure $\epsilon_{s}=\epsilon_{p}>\epsilon_{0}$, but not at $C_{0}=30 \mu \mathrm{M}$. We therefore consider the data obtained at $C_{0} \geqslant 60 \mu \mathrm{M}$ reliable equilibrium data, whereas the two data points at lower $C_{0}$ may represent nonequilibrium island formation.

We plot $C_{0}$ vs $t_{0}$ and fit the inverted function:

$$
\begin{aligned}
C_{0}= & \frac{2 \epsilon_{0} A_{\mathrm{LC}}}{A^{*} V N_{A}} \\
& \times \frac{\left(\lambda_{L p}-2 \lambda_{p s}\right) e^{\lambda_{L p} t_{0}}}{\lambda_{L p}\left(e^{\left(\lambda_{L p}-2 \lambda_{p s}\right) t_{0}}+e^{\lambda_{L p} t_{0}}-2\right)-2 \lambda_{p s}\left(e^{\lambda_{L p} t_{0}}-1\right)},
\end{aligned}
$$

which is extracted from Eq. (5) for $\epsilon_{p}=\epsilon_{0}$ and $t=t_{0}$. While our current data are too scarce to carry out a proper fitting procedure, and as experimental artifacts also require the introduction of small offsets in concentration and time (see Appendix I), we see in Fig. 7 that Eq. (6) reproduces the experimental data well with reasonable parameter values. The formalism thus appears adequate, but future work is required to obtain time-resolved data with sufficient resolution and accuracy to place confidence in the quantitative fitting results. 


\section{DISCUSSION}

An interesting question is whether the topological defects on the originally fully tangential-aligned shells function as seeds of island formation, since $\mathbf{n}(\mathbf{r})$ is highly distorted around the defects to begin with. Indeed, in the low-asymmetry shell in Fig. 1, the first islands to be detected in Fig. 1(d) include islands seeded near a topological defect. However, there are also regions with defects that remain fully tangential-aligned well after islands start appearing in defectfree locations. In fact, due to their extension as line defects [2] from shell inside to outside, the half-integer disclinations that typically populate the pristine shell are best located near the thinnest regions to minimize the free energy [4], and as demonstrated in Fig. 3 this raises the energy cost of forming islands. As a consequence, no formation of islands with hybrid $\mathbf{n}(\mathbf{r})$ is detected near defects in the strongly asymmetric shells in Fig. 2, because all defects are located in the thinnest region, beyond the critical point of the $\left(\epsilon_{p}, \theta\right)$ phase diagram, where $d<K / W$. Any island-promoting impact of the deformed director field around defects is thus effectively canceled by the tendency of defects to be located at the thinnest shell regions, where island formation is particularly costly in terms of elastic energy.

Another peculiarity of LC shells that we believe has more impact is the opposite signs of mean curvature on the insides and outsides. Even though the shell curvature is not strong, a sensitivity to the sign of mean curvature of the interaction between the LC and adsorbing amphiphiles has been confirmed both for low molar mass surfactants $[33,34]$ and for block copolymers [28]. We hypothesize that a corresponding sensitivity to the sign of interface curvature for lipids is one of the main reasons for the surprisingly low value of the stability limit $\epsilon_{0}^{\prime}$ when the primary phase is on the shell inside. Concretely, curvature should affect the effective area $A^{*}$ per lipid molecule, the critical lipid coverage $\epsilon_{c}$ for changing the anchoring from tangential to radial, as well as the Flory interaction parameter $\chi$ for lipids at the LC-water interfaces.

The organization of amphiphiles at a polar-nonpolar interface such as a water-LC interface depends strongly on the relation between the effective sizes of the hydrophilic and hydrophobic moieties, as conveniently described by the critical packing parameter $p_{c}$ [35]. While lipids typically have $p_{c} \approx 1$, promoting a flat interface, small deviations can make a significant difference when comparing interfaces with opposite signs of mean curvature. Cumberland et al. studied the adsorption of DLPC on nematic 8CB at an initially flat interface, surprisingly finding that the interface spontaneously bulged out towards the polar phase (in their case glycerol) as the concentration of lipid was increased [36]. In other words, at high enough coverage fraction, as in a condensed lipid island, DLPC prefers positive mean curvature of an LC boundary onto which it adsorbs, and the authors thus postulated that $p_{c}<1$ under these conditions.

This observation is highly significant for us, as it means that the DLPC adsorption on the outside of LC shells takes place with the preferred sign of mean curvature, whereas DLPC adsorbing on the inside is imposed the wrong sign of mean curvature. If $A^{*}=0.4 \mathrm{~nm}^{2}$ is correct for a flat interface, we may thus expect a somewhat lower value on the shell outside and a somewhat greater value on the shell inside, as less efficient packing can be expected the more the curvature deviates from the preferred one. In our experiments, the difference should be higher for the shells in which we introduced lipid via the inner phase, because these had lower radii and thus greater magnitude of curvature. Since the maximum primary inner phase lipid coverage $\epsilon_{p}^{m}$ according to Eq. (4) is proportional to $A^{*}$, we can conclude that our value obtained with $A^{*}=0.4 \mathrm{~nm}^{2}$ is too low, hence the apparent discrepancy from the expected stability limit $\epsilon_{0}$ is exaggerated.

Moreover, the stability limit $\epsilon_{0}$ should be affected by the different signs of mean curvature, as the interaction of the twin acyl chains per lipid molecule with the bulk LC, and thus the anchoring-determining effect, is favored by the greater conformational freedom provided by adsorption on an LC boundary with negative mean curvature, yielding less crowding. Also the interaction parameter $\chi$ may be affected by the sign of mean curvature of the interface. Finally, we expect the dynamic variation of the lipid coverage to be affected as well, since the opposite signs of mean curvature and the nonequal surface areas should introduce an asymmetry in the rate constants for lipids translating through the shell, $\lambda_{p s} \neq \lambda_{s p}$, in conflict with our approximation. Elucidating the true impact of negative and positive mean curvature of an LC-water interface on the values of $A^{*}, \epsilon_{c}, \chi, \lambda_{p s}$, and $\lambda_{s p}$ is an important objective for future studies. Even without quantitative data on these parameters, the classic understanding of amphiphile adsorption at interfaces generally always having a preferred nonzero curvature [35], and the observations of [36], clearly suggest that there is a significant curvature sensitivity that is not yet captured by our analysis.

One may even contemplate that the LC-aqueous interface may exhibit local variations in curvature, and the LC in between local variations in thickness, within and outside islands. Such effects might be expected to arise from the spatial variations of the director field and corresponding elastic energies, in particular near topological defects within islands and at their perimeter (see Fig. 6), as well as by the preferred curvature of the condensed 2D phases of lipid. Interface curvature modulation has been experimentally detected in glass forming cholesterics [37], at nematic free surfaces in the vicinity of defect-inducing particle inclusions [38], and confirmed by computer simulation for cholesteric shells with in-plane helix orientation [39]. However, the magnitude is in the nanometer range $[37,38]$ and thus too subtle to be detected by the optical microscopy normally employed to study LC shells.

Apart from this impact of curvature on the equilibrium conditions, the much longer time for seeding the first island at the low minimum concentrations of lipid introduced via the inner phase, and the reduced further growth rate compared to the behavior at higher lipid concentrations, suggest that kinetics may play a role. We note that $\epsilon_{p}$ does not grow continuously and evenly around the shell, but it increases in discrete steps, each corresponding to the deposition of a liposome. We should thus take local fluctuations in lipid concentration into account [20], in addition to the global lipid coverage. At intermediate lipid coverage fractions, from $\epsilon_{p} \approx 0.4$ to $\epsilon_{p} \approx 0.6$, the $G^{\prime}$ curves (shown in Fig. 8 for primary phase on the inside) flatten out, yielding a free energy that is nearly independent of lipid coverage in this regime. This means that local fluctuations in 


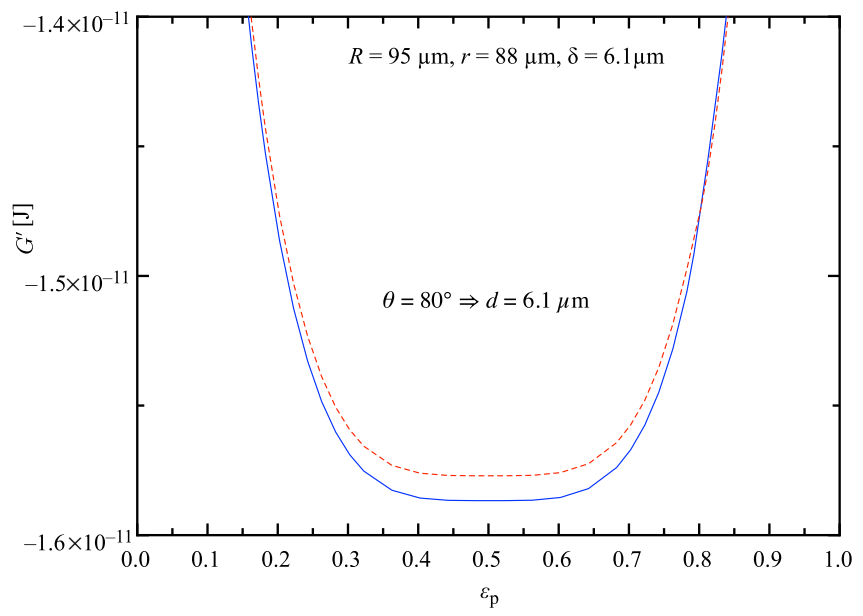

FIG. 8. Free energy as function of lipid surface coverage fraction. Rescaled free energy $G^{\prime}$ for a shell with geometrical parameters as in the experiment of Fig. 16 and primary inside, calculated at $\theta=80^{\circ}$, corresponding approximately to the location where islands were observed in the experiment. The blue fully drawn curve corresponds to the original tangential alignment $\left(\alpha_{p}=90^{\circ}\right)$ whereas the red dashed one corresponds to hybrid alignment $\left(\alpha_{p}=0^{\circ}\right)$. Common parameter values used for calculations: $\epsilon_{s}=0.0001, \alpha_{s}=90^{\circ}, \chi=2, A^{*}=0.4 \mathrm{~nm}^{2}, K=10^{-11} \mathrm{~N}$, $W=20 \mu \mathrm{J} / \mathrm{m}^{2}, B=100, \epsilon_{c}=0.8, T=300 \mathrm{~K}$.

$\epsilon_{p}$ can remain for long times, similar to how critical fluctuations arise near a second-order phase transition due to the very small change in free energy even for large variations in the order parameter. If a new liposome adsorbs at a point of high local $\epsilon_{p}$, the lipid content may transiently increase beyond the stability limit, to $\epsilon_{p}>\epsilon_{0}$. If the LC responds by switching to normal director $\left(\alpha_{p}=0^{\circ}\right)$ on the primary side, an island with hybrid LC has been born. Gibbs' free energy then follows the $\alpha_{p}=0^{\circ}$ curve in Fig. 8, which is lower than before the director reorientation for $\epsilon_{p}>\epsilon_{0}$, hence the island remains, albeit in a metastable state. We thus tentatively propose that phase separation at a global $\epsilon_{p}<\epsilon_{0}$ may be possible due to a kinetically stabilized nonequilibrium state.

The attraction between primary and secondary islands shown in Figs. 5(d)-5(f) constitutes an alternative example of interaction mediated by the elastic forces of a distorted LC, with similarities to the way topological defects interact via nematic elasticity [40,41]. It is instructive to compare the two cases to also reveal the differences. As for islands, $\mathbf{n}(\mathbf{r})$ is deformed in the vicinity of a topological defect, and this deformation is amplified if two defects with the same sign of topological charge are close to each other. The two defects thus repel each other, by the force provided by the deformed director field between them. On the other hand, two defects with opposite sign attract, because their joint impact on $\mathbf{n}(\mathbf{r})$ reduces the overall deformation. Islands on LC shells have no net topological charge [Fig. 1(j)] and the LC elasticitymediated interaction between primary and secondary islands is always attractive, because their overlap always reduces the deformation of $\mathbf{n}(\mathbf{r})$.

While topological defects are often seeded by inclusions $[40,41]$, they can appear in the LC itself, having no material component but comprising simply a singularity in the director field. The attractive motion of two such pure topological defects of opposite sign can be very fast, especially towards the end of the process, as there is no mass transfer involved, only reorientation of the director. In contrast, the attraction of primary and secondary islands is quite slow, as the motion involves lateral transfer of the condensed lipid phases. Moreover, the final step of attraction for opposite-signed topological defects of equal magnitude is complete overlap and annihilation, whereas primary and secondary islands are topologically separated to interfaces with no crossing point, hence the final stage is a locked superposition into an island sandwich. One example of defect interaction should resemble this type of island interaction quite closely, and that is the case when surface defects (boojums) are located on opposite sides of fully tangential-aligned shells $[1,2,4]$. However, to the best of our knowledge, such boojums always arise in pairs, located on top of each other from the start, hence their mutual attraction cannot be experimentally studied in the same way.

Finally, the localization of the islands on the two opposing interfaces means that the strength of attraction depends on where they are on the shell (15). In thick regions, the elastic energy cost of deformation can be so low that the gain from superposition is insignificant, and it may be difficult to detect the island attraction experimentally. In thin regions, in contrast, the removal of director field deformation by superposing adjacent primary and secondary islands into a sandwich reduces the free energy sufficiently to cause significant attraction, as in Figs. 5(d)-5(f). At high enough $C_{0}$, the entire shell eventually ends up normal-aligned on both sides. The trajectory to this end state is complex, governed by 2D lipid phase separation feeding back to bulk LC elasticity and the parallel process of lipid transfer across the LC. Both processes are tuned by the spatially varying $d$ and we thus see island dynamics changing as function of location and time. A full elucidation is a topic for future work, but we note that near the end of the process, before $\mathbf{n}(\mathbf{r})$ becomes uniform, shells always exhibit a ring of hybrid alignment; see Appendix K and Fig. 19.

The ease in getting tangential and normal alignment to coexist on the same surface of an LC shell using lipid phase separation at the interface, and the possibility to control the overall pattern and its development dynamics by tuning the lipid concentration, shell asymmetry and reaction time, can be very powerful from the point of view of advanced materials science. By polymerizing the shell a certain time after exposure to liposomes, when a desired pattern has developed, the self-assembled structure can be frozen in [42]. This would allow programming specific types of shape morphing, including buckling, opening and closing, of LC elastomer shells [14-16] by creating complex patterns of tangential, hybrid, and normal director fields via lipid phase separation prior to cross-linking the precursor. Equally interesting are the possibilities to create patchy colloidal particles with nontrivial van der Waals interaction symmetries, extending the recent work of Fuster et al. based on polymerized LC microdroplets [17]. The dynamic formation, growth, and interactions of lipid islands would allow more complex patterning, and the use of shells - which can be produced in colloidal scale using tip streaming [43] — gives access for such patterning to surfaces 
(a)

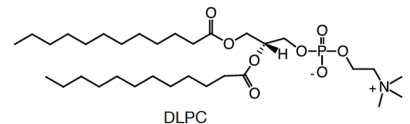

(c)

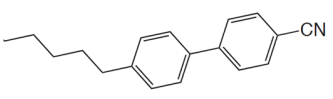

$5 \mathrm{CB}$

(b)

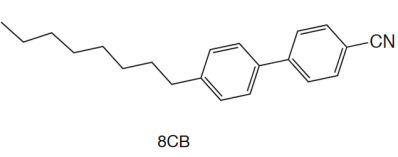

FIG. 9. Chemical structures. Molecules of (a) DLPC, (b) 8CB, (c) 5CB), and (d) PVA ( $88 \%$ hydrolyzed).

with negative mean curvature. Since the polymerized shells can be harvested as spheres or collapsed into bowls [42], they could become a rich toolbox for colloidal self-assembly research.

A further stimulating extension would be to study the adsorption of mixed lipid vesicles or even actual cell membranes on LC shells, to investigate how the presence of different lipid types and membrane proteins affects the phase diagram and consequent phase separation in monolayers developing on hydrophobic liquid substrates. Indeed, while our investigations here were limited to DLPC, chosen in order to allow direct comparisons with related prior work, other amphiphilic molecules can undergo 2D phase separation on LCs, provided that their interaction parameter $\chi$ is in a suitable range. Even common ionic surfactants such as sodium dodecyl sulfate or cetyl trimethylammonium bromide can be used if their mutual head group repulsion is suppressed by salt addition [44], and the impact of regular zwitterionic lipids should depend on the $\mathrm{pH}$ of the aqueous phases. While liposome adsorption into supported lipid bilayers on hard hydrophilic substrates is well understood, the corresponding adsorption into monolayers on soft hydrophobic substrates has only recently come into attention. Among the phenomena identified are Laplace pressure-driven traction stress [45], transient Marangoni flows [20] and a spreading process modified by the solubilization of the outer leaf of the liposome into the substrate [19]. LC shells may provide complementary information, as they allow the study of naturally occurring lipids in standard optical microscopy, without fluorescent labeling, and as the secondary island formation directly reports on lipid solubilization and transfer through the LC.

\section{MATERIALS AND METHODS}

DLPC (1,2-dilauroyl-sn-glycero-3-phospho-choline) was purchased from Avanti Polar Lipids. The LCs 5CB (4cyano-4'-pentylbiphenyl, phase sequence $\mathrm{Cr}$. $24.0 \mathrm{~N} 35.6$ iso. $/{ }^{\circ} \mathrm{C}$ ) and $8 \mathrm{CB}$ (4-cyano-4'-octylbiphenyl, Cr. $21.5^{\circ} \mathrm{C}$ $\mathrm{SmA} 33^{\circ} \mathrm{C} \mathrm{N} 40.5$ iso. $/{ }^{\circ} \mathrm{C}$ ) were purchased from Synthon Chemicals. Throughout the experiments, we used 0-1 wt $\%$ PVA $\left(M_{\mathrm{w}}=13-023 \mathrm{~kg} / \mathrm{mol}, 87 \%-89 \%\right.$ hydrolyzed $)$ from Sigma-Aldrich, dissolved in deionized water (resistivity $18 \mathrm{M} \Omega / \mathrm{cm}$, Sartorius arium pro-DI). Texas Red-DHPE for fluorescence microscopy was bought from Thermo Fisher Scientific. Chemical structures of DLPC, 5CB, 8CB, and PVA are shown in Fig. 9.
We produced shells with the LC heated to the isotropic phase $\left(40^{\circ} \mathrm{C}\right.$ for $5 \mathrm{CB}$ and $50^{\circ} \mathrm{C}$ for $\left.8 \mathrm{CB}\right)$ with aqueous PVA solution $(1 \mathrm{wt} \%)$ as outer and inner phase using a coaxial glass capillary microfluidic setup, as described by Weitz and co-workers [46]. We collected the shells in a glass vial and stored in an incubator at $30^{\circ} \mathrm{C}$.

To prepare a solution of liposomes (giant unilamellar vesicles), we followed the method described by Peng et al. [47]. We first dissolved DLPC $(0.93 \mathrm{mg})$ in dichloromethane (DCM) $(0.2 \mathrm{ml})$ in a vial, then evaporated the DCM to form a dry layer of DLPC on the inside of the vial. We then added DI water and tip-sonicated the sample (Dr. Hielscher UP200St, $10 \mathrm{~W}$ power and $30 \%$ amplitude) to make $3 \mathrm{ml}$ of liposome stock solution $(0.5 \mathrm{mM}$, characterization by dynamic light scattering in Appendix L and Fig. 20), kept at $4{ }^{\circ} \mathrm{C}$ until use. The $\mathrm{pH}$ value of pure DI water was measured to be 5 and the addition of DLPC gave rise to no considerable change. The stock solution was diluted as required to obtain a liposome solution with desired concentration. Liposomes were added to the outer phase by a 1:1 mixing (by volume, $0.5 \mathrm{ml}$ of each) of liposome solution (stock solution or diluted as required) and shell emulsion (8CB shells in SmA phase, 5CB shells in nematic phase). Because the liposome solution contains no PVA, the final PVA concentration in the outer phase during such lipid adsorption experiments is $0.5 \mathrm{wt} \%$.

After adding liposome solution (outer phase is primary phase) or directly after shell production (inner phase is primary phase), we filled the emulsion with shells (at room temperature) into a rectangular glass capillary of dimensions $0.3 \mathrm{~mm} \times 3 \mathrm{~mm} \times \sim 30 \mathrm{~mm}$. For temperature control, we placed the capillaries in a Linkam T95-PE hot stage mounted on a polarizing optical microscope (POM; Nikon Eclipse LV100ND). Videos and images were captured by a Sony FDR AX33 camcorder, mounted on the POM. Fluorescence microscopy experiments on samples in which the liposome solution contained fluorescently labeled lipids (see Appendix A) were done using a Nikon Eclipse Ti2 inverted microscope equipped with a TRITC-B filter (wavelength range: $525-556 \mathrm{~nm}$ ) and a Pixelink camera.

All data needed to evaluate the conclusions are publicly available at Ref. [48].

\section{ACKNOWLEDGMENTS}

We thank E. Anyfantakis for help with the fluorescence microscopy and L. W. Honaker for advice on preparing the liposome solution. Financial support from the European Research Council under the European Union H2020 Programme, Grant Agreement No. 648763 (consolidator project INTERACT) is gratefully acknowledged. A.S. acknowledges support from the Fonds National de la Recherche (FNR, doctoral training grant PRIDE MASSENA, grant code 2016/10935404). D.G. is supported by the "Excellence Project 2018" of the Cariparo Foundation.

A.S. carried out all experiments and contributed to the analysis of results. J.P.F.L coordinated the analysis and the writing of the paper. D.G., G.S., and J.P.F.L performed analytical calculations. All authors contributed to the manuscript. The authors declare no competing interests. 

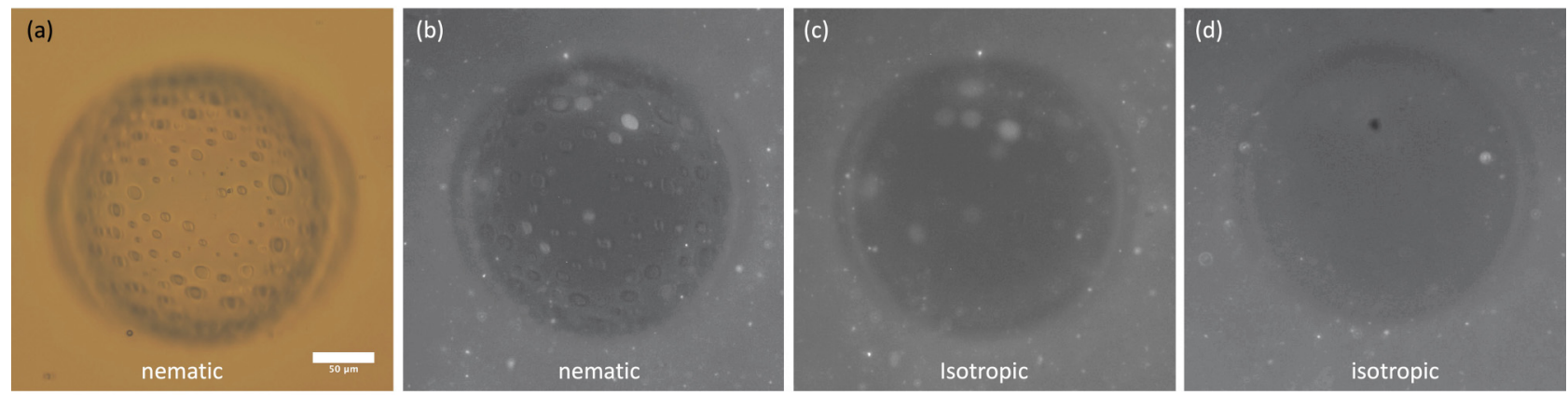

FIG. 10. Fluorescence microscopy confirmation of lipid island formation. Images of a 5CB shell with $0.12 \mathrm{mM}$ lipids in the outer phase, consisting of DLPC with $0.1 \mathrm{~mol} \%$ Texas-Red-labeled DHPE. (a). Bright field image of the nematic $\left(28^{\circ} \mathrm{C}\right)$ shell showing islands on the thicker side of the shell. (b). The corresponding fluorescence microscopy image shows bright gray spots on the shell confirming that islands have higher lipid coverage fraction $\epsilon$. (c). We heat the shell to the isotropic phase and still see islands on the shell immediately after the phase transition. (d). Keeping the shell at $37^{\circ} \mathrm{C}$ for 5-8 min yields an island-free shell. Scale bar is $50 \mu \mathrm{m}$.

\section{APPENDIX A: CONFIRMATIONS OF LIPID ISLAND FORMATION BY FLUORESCENCE MICROSCOPY}

To confirm that the islands seen in POM indeed correspond to local regions of condensed lipid phase we conduct an experiment on $5 \mathrm{CB}$ shells exposed to a liposome solution where $0.1 \mathrm{~mol} \%$ of fluorescently labeled Texas Red-DHPE has been mixed with DLPC. After adding the liposomes to the outer phase, at a concentration yielding an initial lipid concentration $C_{0}=0.12 \mathrm{mM}$, we see primary island formation after about 10 min. After additional 10-12 min, the shells are transferred to a pure water bath to wash out excess Texas Red DHPE in the outer phase. The emulsion is then immediately filled into a capillary for fluorescence microscopy, the results shown in Fig. 10.

We first observe the shells in the nematic phase, Fig. 10(a) showing a bright-field image where many small islands are easily visible. The corresponding fluorescence microscopy image is shown in Fig. 10(b), showing roughly the same pattern of islands (some island motion and additional island generation takes place while switching between microscopy modes), here recognized as brighter spots on a darker background. This reflects the increased fluorescence intensity from the islands due to their higher lipid concentration. However, as the difference in the primary interface lipid coverage $\epsilon_{p}$ within and outside islands is not very large, the contrast is weak, requiring digital contrast enhancement in the software Image $\mathrm{J}$ to detect the islands. The islands are uniformly bright, confirming that islands are uniformly enriched with lipids. Bright regions in the surroundings show that the washing stage was not sufficient to remove all liposomes from the outer phase.

Upon heating to the isotropic phase and waiting for a few minutes [Fig. 10(d)] nearly all islands are gone, with a uniform fluorescence reflecting an even distribution of lipids across the entire shell. This confirms that phase separation requires the interaction with the nematic LC phase in the shell. However, fluorescence imaging immediately after heating to isotropic [Fig. 10(c)] still shows islands, confirming that the redistribution of lipids from the phase separated state takes some time. In brightfield microscopy (see Supplemental Video 1 [21]), in contrast, the islands become invisible immediately after the shell turns isotropic. If the shell is recooled to nematic without isotropic annealing, the islands reappear at identical sizes and locations also in brightfield microscopy. This demonstrates the power of the LC in visualizing lipid condensation, requiring no labeling.

\section{APPENDIX B: CONFIRMATION OF ISLAND ORIENTATION WITH RESPECT TO THE SURROUNDING DIRECTOR FIELD}

In order to confirm that the spindle-shaped small islands orient with their long axis along $\mathbf{n}\left(\mathbf{r}_{\mathbf{o}}\right)$, we temporarily cool nematic $8 \mathrm{CB}$ shells [Fig. 11(a)] to $30^{\circ} \mathrm{C}$, such that they transition into the smectic-A (SmA) phase [Fig. 11(b)]. The nonzero shell thickness imposes a geometrical frustration on the smectic layers as they form in the curved shell, leading to an undulation instability visualized as stripes running along the director $[49,50]$. This allows us to unambiguously determine $\mathbf{n}\left(\mathbf{r}_{\mathbf{o}}\right)$ throughout the shell, and, indeed, we see in Fig. 11(b) that the shell's primary islands are elongated along $\mathbf{n}\left(\mathbf{r}_{\mathbf{o}}\right)$.
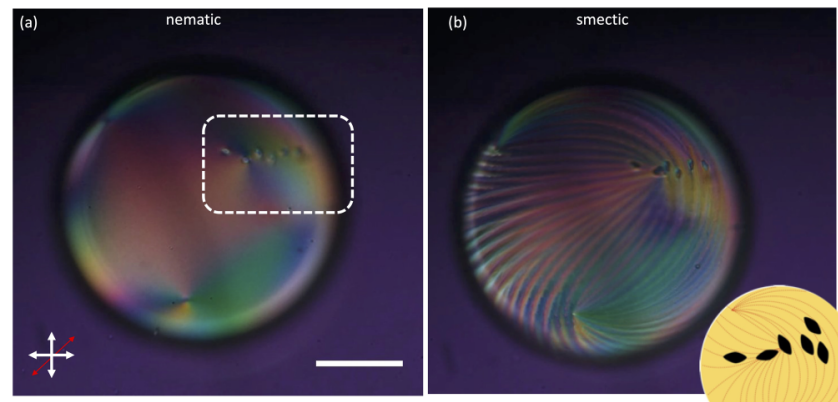

FIG. 11. Confirmation of island orientation. (a) A snapshot of an $8 \mathrm{CB}$ LC shell in the nematic $\left(34.4^{\circ} \mathrm{C}\right)$ phase just after the birth of primary islands (within the white dotted rectangular box). (b) Cooling to the $\mathrm{SmA}$ phase $\left(30^{\circ} \mathrm{C}\right)$ triggers an undulation instability with stripes appearing along the director, confirming that islands are aligned with their long axes along $\mathbf{n}\left(\mathbf{r}_{\mathbf{o}}\right)$. The inset gives a schematic representation of the island alignment with respect to $\mathbf{n}\left(\mathbf{r}_{\mathbf{o}}\right)$. Both images are taken with $\lambda$ plate (red double arrow) inserted between crossed polarizers (white double arrows). Scale bar represents $50 \mu \mathrm{m}$. 


\section{APPENDIX C: ABSENCE OF ISLAND FORMATION IN THE THINNEST REGION OF HIGHLY ASYMMETRIC SHELLS}

In highly asymmetric shells, the elastic energy penalty of primary island formation is so high that no islands form in a region around the thinnest point, defined by a limiting value of the polar angle $\theta$ [see Figs. 1(b) and 2]. This is seen very clearly by speeding up a video of island formation in a highly asymmetric shell, as in Supplemental Video 3 [21]. (Fig. 2 shows three frames from this video). These are $5 \mathrm{CB}$ shells with a very thin top, around which all topological defects are gathered, as easily recognized in the brush-rich texture. Inside a circular boundary shown as a green dashed ellipse (the elliptical appearance is due to an optical artifact explained in [28]) in Fig. 2, no islands are seen at any point in the video, while islands born just outside the boundary rapidly move outwards and downwards, towards thicker regions of the shell to reduce the elastic energy penalty. The green dashed boundary thus corresponds to the critical point of the $\left(\epsilon_{p}, \theta\right)$ phase diagram, as discussed in the main text and below.

To find an approximate value of the polar angle $\theta$ for this boundary, we first measure the distance between the center and the boundary along the semiminor axis. In Fig. 2 we find this distance to be $s \approx 46.6 \mu \mathrm{m}$. The outer radius of the shell is $103 \mu \mathrm{m}$. We can thus estimate the polar angle of the critical point through

$$
\tan \theta=s / r=0.45 \Rightarrow \theta=\arctan 0.45=0.42=24^{\circ} .
$$

Considering the difficulties in accurately measuring distances in Fig. 2, due to the optical artifact [28], we can conclude that the polar angle is in the range of $\theta \approx 25^{\circ}$ at the critical point in these shells.

\section{APPENDIX D: DERIVATION OF FREE ENERGY DENSITY}

We build on Gupta et al. [7] in deriving Eq. (2) for the free energy density, but we extend the model to the situation of LC shells, where the existence of two spherical interfaces with different area and a location-dependent thickness, independently varying lipid coverage at each interface, as well as the freedom of the director to independently change orientation at either interface constitute major differences. These differences require significant modifications, as elaborated in the following. Like [7], we use standard Flory-Huggins thermodynamics of mixing formalism [51] for the contribution to the free energy from the distribution of lipids at one entire LC-aqueous interface to the free energy:

$$
G_{\mathrm{mix}}=k T N[\epsilon \ln \epsilon+(1-\epsilon) \ln (1-\epsilon)+\chi \epsilon(1-\epsilon)],
$$

where $k$ is Boltzmann's constant, $T$ is the absolute temperature, $N$ is the total number of lipid adsorption "sites" within the area considered, $\epsilon$ is the lipid coverage fraction at the relevant interface, and $\chi$ is the Flory interaction parameter, here parametrizing the tendency of lipids to cluster together (higher value means stronger clustering tendency). The first two terms in Eq. (D1) are entropic whereas the final one is enthalpic.

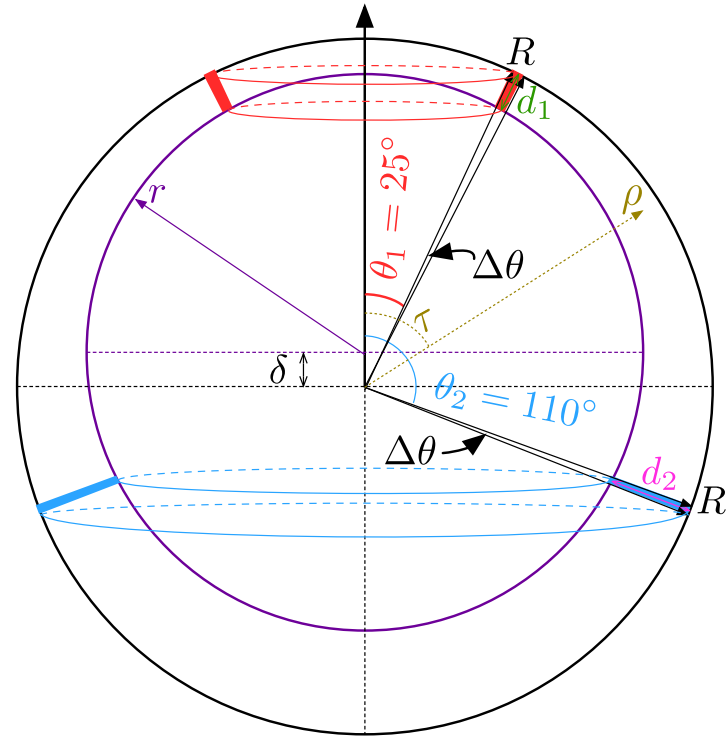

FIG. 12. Illustrations of the parameters and geometrical features introduced when integrating the free energy densities. The spherical outer shell interface has radius $R$, and the inner droplet has radius $r$, but the latter is from the inner droplet center, which is shifted vertically by the distance $\delta$ from the outer shell center. We take the latter as the overall geometrical origin in the analysis. At any polar angle $\theta$ (here two cases, $\theta_{1}=25^{\circ}$ and $\theta_{2}=110^{\circ}$ are shown as illustration) we define a narrow conical cross section with the shell in which we consider the free energy constant. This is acceptable as we consider the cone angle variation $\Delta \theta$ very small; in the drawing it is greatly exaggerated for clarity. The two example cross sections are highlighted in red $\left(\theta_{1}=25^{\circ}\right)$ and blue $\left(\theta_{2}=110^{\circ}\right)$, respectively. We introduce the help variables $\rho$ and $\tau$ to parametrize arbitrary distances from the origin and arbitrary polar angles, respectively, during integration. To obtain the surface energy terms we integrate from $\tau=\theta$ to $\tau=\theta+\Delta \theta$ over a ring at $\rho=R$ for the outer LC-aqueous interface and at $\rho=(R-d)$ for the inner interface. For the volume integral (elastic energy contribution) we integrate from $\rho=(R-d)$ to $\rho=R$ and from $\tau=\theta$ to $\tau=\theta+\Delta \theta$. Since the problem has full rotational symmetry about the vertical axis, the contribution from the azimuthal integration is simply $2 \pi \rho \sin \tau$ for the surface as well as the volume integrals.

Because we have full rotational symmetry about the vertical center axis of a shell, $\theta=0$ to $\theta=\pi$, i.e., the azimuthal angle $\phi$ does not enter the free energy, we consider the energy within a conical cross section at polar angle $\theta$ with variation $\Delta \theta$, as illustrated in Fig. 12. The areas of its two interfaces with water can be calculated, introducing $\rho$ as the relevant distance from the origin, which we take as the center of the shell (center of the outer sphere), and the integration variable $\tau$ for parametrizing the variation in $\theta$, as

$$
\begin{aligned}
A_{\text {interface }} & =\int_{\theta}^{\theta+\Delta \theta} 2 \pi \rho \sin \tau \rho d \tau \\
& =2 \pi \rho^{2}[\cos \theta-\cos (\theta+\Delta \theta)] \\
& \approx 2 \pi \rho^{2}[\cos \theta-(\cos \theta-\Delta \theta \sin \theta)] \\
& =2 \pi \rho^{2} \sin \theta \Delta \theta .
\end{aligned}
$$




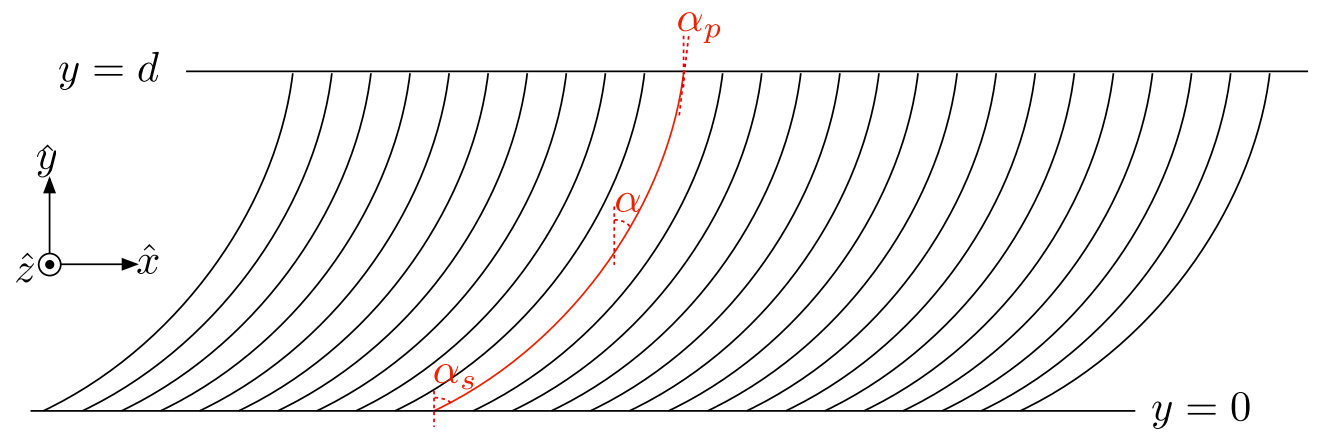

FIG. 13. Parametrizing the bent director field. We use the angle $\alpha$ to describe the director orientation in the $x-y$ plane when parametrizing the director field, bending from the director orientation $\alpha_{s}$ at the secondary to $\alpha_{p}$ at the primary side.

Since $\Delta \theta$ is very small, we have used the trigonometric approximation $\cos (\alpha+\beta) \approx \cos \alpha-\beta \sin \alpha$ valid for small $\beta$. For the outer interface, $\rho_{\text {out }}=R$ and for the inner interface, $\rho_{\text {in }}=R-d$, where $d$ is the thickness of the shell at polar angle $\theta$, as defined and calculated in the following section. Note that these definitions break down in case of a very small inner droplet, $r<R / 2$, where the origin would be in the LC phase. However, such extremely thick shells are not part of our study, hence this limitation causes no problems within the scope of the paper.

Considering that one lipid at maximum coverage $(\epsilon=1)$ takes up the area $A^{*}$, the total number of lipid adsorption sites on the inner and outer interfaces of such a conical cross section can then be written

$$
N=\frac{A_{\text {interface }}}{A^{*}}=\frac{2 \pi \rho^{2} \sin \theta \Delta \theta}{A^{*}} .
$$

This gives us the Flory-Huggins contributions for interface $i$ [primary $(p)$ or secondary $(s)$ ] of the conical cross section:

$G_{\text {mix }}^{i}=\frac{\Gamma k T}{A^{*}} \rho^{2}\left[\epsilon_{i} \ln \epsilon_{i}+\left(1-\epsilon_{i}\right) \ln \left(1-\epsilon_{i}\right)+\chi \epsilon_{i}\left(1-\epsilon_{i}\right)\right]$,

where we introduced

$$
\Gamma=2 \pi \sin \theta \Delta \theta .
$$

When an interface is the primary one, $\epsilon_{i}=\epsilon_{p}$, while for the secondary interface, $\epsilon_{i}=\epsilon_{s}$.

Next, we consider the liquid crystal-specific contributions to $G$, which are the elastic bulk contribution and the anchoring surface contributions on inside and outside. The first here corresponds to a combination of splay and bend, as can be concluded by parametrizing the director field mediating between the director anchoring $\alpha_{s}$ and $\alpha_{p}$ at the secondary and primary shell interfaces, respectively; see Fig. 13. Ignoring thermal fluctuations as well as the impact of the shell curvature, which is negligible in our case since we have assumed $R \gg(R-r)$, we can describe the director as

$$
\mathbf{n}=(\cos \alpha, \sin \alpha, 0),
$$

where the director orientation angle $\alpha$ is parametrized as a linear function (see Fig. 13 for coordinate definitions):

$$
\alpha=\alpha_{s}+\frac{y}{d}\left(\alpha_{p}-\alpha_{s}\right) .
$$

In applying Eq. (1) to this director field, we first calculate the divergence and curl of $\mathbf{n}$ :

$$
\begin{gathered}
\nabla \cdot \mathbf{n}=\cos \alpha \frac{\alpha_{p}-\alpha_{s}}{d}, \\
\nabla \times \mathbf{n}=\hat{z} \sin \alpha \frac{\alpha_{p}-\alpha_{s}}{d} .
\end{gathered}
$$

Since $\mathbf{n}$ is in the $\hat{x}-\hat{y}$ plane, we immediately see that the twist term in Eq. (1) is zero, as expected. Calculating the remaining terms in full, we obtain

$$
g_{e}=\frac{1}{2} K_{1} \cos ^{2} \alpha \frac{\left(\alpha_{p}-\alpha_{s}\right)^{2}}{d^{2}}+\frac{1}{2} K_{3} \sin ^{2} \alpha \frac{\left(\alpha_{p}-\alpha_{s}\right)^{2}}{d^{2}} .
$$

By approximating $K_{1} \approx K_{3} \approx K$, we obtain

$$
g_{e}=\frac{1}{2} K \frac{\left(\alpha_{p}-\alpha_{s}\right)^{2}}{d^{2}} .
$$

Because $K_{3} \approx 1.6 K_{1}$ [27], this overestimates the elastic contribution. However, within the overall approximations done in our analysis, it is sufficient to work with correct orders of magnitude, hence $K \approx K_{3}$ is acceptable.

We need to integrate $g_{e}$ over the volume of the conical cross section to get the elastic free energy contribution, and to this end we use the same trigonometric approximation as in Eq. (D2), since $\Delta \theta$ is very small:

$$
\begin{aligned}
G_{\text {elast }} & =\frac{K\left(\alpha_{p}-\alpha_{s}\right)^{2}}{2 d^{2}} \int d V \\
& =\frac{K\left(\alpha_{p}-\alpha_{s}\right)^{2}}{2 d^{2}} \int_{R-d}^{R} \int_{\theta}^{\theta+\Delta \theta} 2 \pi \rho \sin \tau \rho d \rho d \tau \approx \\
& \approx \frac{K\left(\alpha_{p}-\alpha_{s}\right)^{2}}{2 d^{2}} \frac{2 \pi \sin \theta \Delta \theta}{3}\left[R^{3}-(R-d)^{3}\right] \\
& =\frac{K \Gamma\left(\alpha_{p}-\alpha_{s}\right)^{2}}{6 d^{2}}\left[R^{3}-(R-d)^{3}\right] .
\end{aligned}
$$

For the anchoring contribution, finally, the form used in [7] for the areal energy density is perfectly adequate for us, but we need to consider it at each interface:

$$
g_{\text {anchoring }}^{i}=\frac{1}{2} W \sin ^{2}\left(\alpha_{i}-\sigma_{i}\left(\epsilon_{i}\right)\right)=\frac{W}{2} \sin ^{2} \Delta \alpha_{i},
$$


where

$$
\sigma_{i}\left(\epsilon_{i}\right)=\frac{\pi}{2}\left[1-\frac{1}{1+e^{-B\left(\epsilon_{i}-\epsilon_{c}\right)}}\right] .
$$

The sigmoidal function $\sigma_{i}(\epsilon)$ switches suddenly, with a slope proportional to the parameter $B$, from $\pi / 2$ at $\epsilon_{i}<\epsilon_{c}$ to 0 at $\epsilon_{i}>\epsilon_{c}$, reflecting the anchoring at an aqueous interface with little lipid coverage to promote tangential alignment $(\alpha=\pi / 2)$, as opposed to an interface with lipid coverage at or above $\epsilon_{c}$, promoting normal alignment $(\alpha=0)$. Equation (D13) then gives the energetic penalty of the director at an interface not adopting the promoted alignment, which amounts to $W / 2$, explaining why we consider $W$ the anchoring strength (assumed identical on both interfaces). When $\mathbf{n}$ follows the anchoring, $\Delta \alpha_{i}=0$ and the anchoring term Eq. (D13) is zero.
We now need to integrate Eq. (D13) over the interfaces of our conical cross section. Using Eq. (D2) and considering both interfaces at once we obtain

$$
\begin{aligned}
G_{\text {anchoring }} & =\pi \sin \theta \Delta \theta W\left[(R-d)^{2} \sin ^{2} \Delta \alpha_{\text {in }}+R^{2} \sin ^{2} \Delta \alpha_{\text {out }}\right] \\
& =\frac{\Gamma}{2} W\left[(R-d)^{2} \sin ^{2} \Delta \alpha_{\text {in }}+R^{2} \sin ^{2} \Delta \alpha_{\text {out }}\right] . \quad \text { (D15) }
\end{aligned}
$$

When we add lipids to the outer phase, $\Delta \alpha_{o u t}=\Delta \alpha_{p}$ and $\Delta \alpha_{i n}=\Delta \alpha_{s}$, and vice versa when the lipids are introduced to the inner phase.

Adding all terms, we get the expressions for the total free energy contribution within the conical cross section under consideration. With the outside as primary side, this reads

$$
\begin{aligned}
G(@ \text { outside })= & \Gamma\left(\frac { k T } { A ^ { * } } \left\{R^{2}\left[\epsilon_{p} \ln \epsilon_{p}+\left(1-\epsilon_{p}\right) \ln \left(1-\epsilon_{p}\right)+\chi \epsilon_{p}\left(1-\epsilon_{p}\right)\right]\right.\right. \\
& \left.+(R-d)^{2}\left[\epsilon_{s} \ln \epsilon_{s}+\left(1-\epsilon_{s}\right) \ln \left(1-\epsilon_{s}\right)+\chi \epsilon_{s}\left(1-\epsilon_{s}\right)\right]\right\} \\
& \left.+\frac{K\left(\alpha_{p}-\alpha_{s}\right)^{2}}{6 d^{2}}\left[R^{3}-(R-d)^{3}\right]+\frac{W}{2}\left[(R-d)^{2} \sin ^{2} \Delta \alpha_{s}+R^{2} \sin ^{2} \Delta \alpha_{p}\right]\right) .
\end{aligned}
$$

In order to remove the dependence on $\Delta \theta$ we consider the rescaled energy:

$$
G^{\prime}=\frac{G}{\Gamma},
$$

which yields our Eq. (2). To facilitate comparisons between different values of $\theta$, which yield differently large conical cross sections, we consider the difference in the free energy contributions between the original tangential and the (potentially) lipidinduced hybrid configurations, relating the difference to the magnitude of the former:

$$
\Delta_{p}=\frac{G^{\prime}\left(\alpha_{p}=0^{\circ}\right)-G^{\prime}\left(\alpha_{p}=90^{\circ}\right)}{\left|G^{\prime}\left(\alpha_{p}=90^{\circ}\right)\right|},
$$

which is what we plot in Fig. 3.

When the lipids are introduced on the inside, then this is the primary side, and we obtain

$$
\begin{aligned}
G(@ \text { inside })= & \Gamma\left(\frac { k T } { A ^ { * } } \left\{(R-d)^{2}\left[\epsilon_{p} \ln \epsilon_{p}+\left(1-\epsilon_{p}\right) \ln \left(1-\epsilon_{p}\right)+\chi \epsilon_{p}\left(1-\epsilon_{p}\right)\right]\right.\right. \\
& \left.+R^{2}\left[\epsilon_{s} \ln \epsilon_{s}+\left(1-\epsilon_{s}\right) \ln \left(1-\epsilon_{s}\right)+\chi \epsilon_{s}\left(1-\epsilon_{s}\right)\right]\right\} \\
& \left.+\frac{K\left(\alpha_{p}-\alpha_{s}\right)^{2}}{6 d^{2}}\left[R^{3}-(R-d)^{3}\right]+\frac{W}{2}\left[R^{2} \sin ^{2} \Delta \alpha_{s}+(R-d)^{2} \sin ^{2} \Delta \alpha_{p}\right]\right),
\end{aligned}
$$

with a corresponding change to $G^{\prime}$ and a formal change $\Delta_{p} \rightarrow$ $\Delta_{s}$.

To obtain the plots in Fig. 3 and Fig. 15, we use $\chi=2$ and $\epsilon_{c}=0.8$ as proposed by Gupta $e t a l ., K \approx 10 \mathrm{pN}$ as typical of $K_{3}$ of 5CB at room temperature [27], a low anchoring strength $W=20 \mu \mathrm{Jm}^{-2}$, and $B=100$.

\section{APPENDIX E: PARAMETRIZATION OF A GENERIC SHELL GEOMETRY}

As defined graphically in Fig. 1(b) for a thin-bottomed shell and here in Fig. 14 for a thin-topped shell, we call the outer shell radius $R$, and the radius of the inner aqueous droplet $r$. We let the origin be the center of the circle defined by the outer shell boundary, and $\hat{x}$ and $\hat{y}$ the horizontal and vertical directions, respectively. The center of the inner droplet is displaced by a distance $\delta$ up or down along $\hat{y}$ depending on the sign of density mismatch. We wish to determine the thickness $d$, defined as the distance along the outer radius from the shell outside until the interface with the inner droplet, as a function of the polar angle $\theta$ of the point under consideration, $x_{o}, y_{o}$, on the shell outside. Because of the cylindrical symmetry of the problem, $d$ is constant around any horizontal circle.

We first translate the equation of the inner droplet boundary from its expression in the inner droplet coordinate system, 


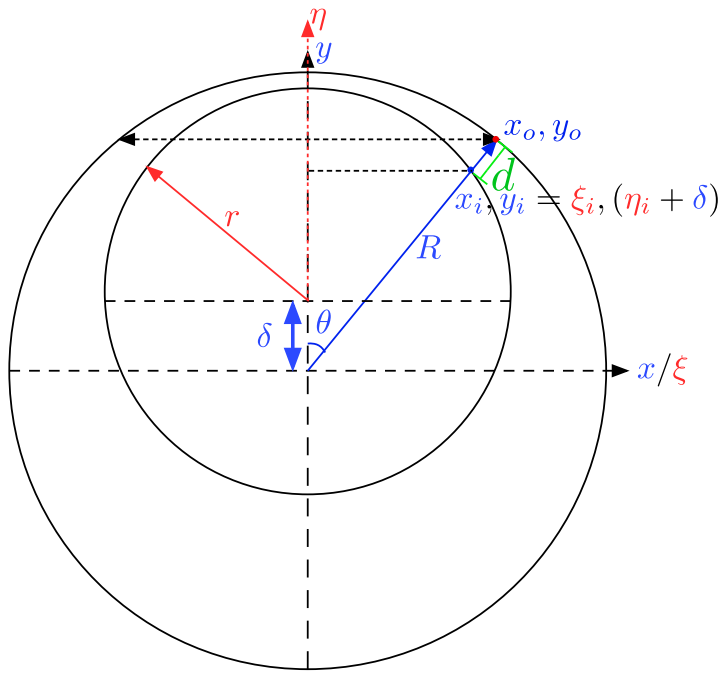

FIG. 14. Parameters used to parametrize the thickness of the shell at arbitrary polar angle $\theta$. This is the thin-topped equivalent of Fig. 1(b). Features in red refer to the $\xi, \eta$ coordinate system with origin at the center of the inner droplet, features in blue refer to the main $x, y$ coordinate system, with origin at the center of the shell, i.e., the center of the outer sphere.

$(\xi, \eta)$, to the main $(x, y)$ coordinate system:

$$
\begin{aligned}
\xi_{i}^{2}+\eta_{i}^{2} & =r^{2} \Leftrightarrow x_{i}^{2}+\left(y_{i}-\delta\right)^{2}=x_{i}^{2}+y_{i}^{2}-2 y_{i} \delta+\delta^{2}=r^{2}, \\
x_{i} & =y_{i} \tan \theta \Rightarrow y_{i}^{2} \tan ^{2} \theta+y_{i}^{2}+\delta^{2}-2 y_{i} \delta-r^{2}=0,
\end{aligned}
$$

where we in the last step brought all terms to one side. In what follows, we use the following identities:

$$
\begin{aligned}
\tan ^{2} \theta+1 & =\frac{\sin ^{2} \theta}{\cos ^{2} \theta}+1=\frac{\sin ^{2} \theta+\cos ^{2} \theta}{\cos ^{2} \theta} \\
= & \frac{1}{\cos ^{2} \theta} \\
= & \sec ^{2} \theta, \\
\Rightarrow y_{i}^{2} \sec ^{2} \theta & -2 y_{i} \delta+\delta^{2}-r^{2}=0, \\
\Rightarrow y_{i}^{2}-2 y_{i} \delta & \cos ^{2} \theta+\left(\delta^{2}-r^{2}\right) \cos ^{2} \theta=0 .
\end{aligned}
$$

The above equation is a quadratic equation, and its roots are

$$
\begin{aligned}
\Rightarrow y_{i} & =\delta \cos ^{2} \theta \pm \sqrt{\delta^{2} \cos ^{4} \theta-\left(\delta^{2}-r^{2}\right) \cos ^{2} \theta} \\
& =\delta \cos ^{2} \theta \pm \sqrt{\delta^{2} \cos ^{2} \theta\left(\cos ^{2} \theta-1\right)+r^{2} \cos ^{2} \theta} \\
& =\cos \theta\left[\delta \cos \theta \pm \sqrt{r^{2}-\delta^{2} \sin ^{2} \theta}\right] .
\end{aligned}
$$

By congruence of triangles, we calculate the vertical distance:

$y_{o}-y_{i}=R \cos \theta-y_{i}=d \cos \theta \Rightarrow d=R-\frac{y_{i}}{\cos \theta}$.

Now we insert Eq. (E4) for $y_{i}$ :

$$
d=R-\delta \cos \theta \pm \sqrt{r^{2}-\delta^{2} \sin ^{2} \theta} .
$$

The correct solution is the negative branch for the square root since the distance $d$ has to be nonnegative, i.e., $d \geqslant 0$.

\section{APPENDIX F: FINDING THE CRITICAL POINT IN THE $\left(\epsilon_{p}, \theta\right)$ PHASE DIAGRAM}

This section is dedicated to obtaining the phase diagram and the critical point using the free energy given in Eq. (2). From Fig. 3(d) we can see that on increasing the value of $\theta$, the normalized difference between the free energy of $\alpha_{p}=0$ and $\alpha_{p}=\pi / 2$ curves starts decreasing for a given $\alpha_{s}=\pi / 2$. Specifically, for some $\theta$ and $\epsilon$, these two curves intersect each other. Therefore, such intersections give us the phase boundary to distinguish the region where islands form to that where they cannot. The equation of the phase boundary, considering primary outside (i.e., $\alpha_{s}=\pi / 2$ ), is given by

$$
\left.G^{\prime}\right|_{\alpha_{p}=0}=\left.\bar{G}^{\prime}\right|_{\alpha_{p}=\pi / 2},
$$

where $G^{\prime}$ is given in Eq. (D17).

Solving Eq. (F1), we arrive at

$$
\frac{d^{2} \cos \left[2 \sigma_{p}\left(\epsilon_{p}\right)\right]}{R^{3}-(R-d)^{3}}=\frac{\pi^{2} K}{12 W R^{2}},
$$

where $d$ is the thickness given in Eq. (E6), depending on $\theta$, and $\sigma_{p}\left(\epsilon_{p}\right)$ is given by Eq. (D14) for $i=p$.

We stress that the equation of contour $(\mathrm{F} 2)$ is an exact expression and it does not depend on $\epsilon_{s}, \chi, T$, and $A^{*}$.

Therefore, using Eq. (F2), we construct the phase diagram in the $\left(\epsilon_{p}, \theta\right)$ plane as shown in Fig. 4, where the filled region corresponds to islands and unfilled one indicates the region with original tangential configuration. Furthermore, we also investigate the value of $\theta$ above which both curves merge with each other. Unfortunately, the analytical or numerical computation of such a point is not illuminating to us. Nonetheless, we give the approximate value where the contour in the figure starts becoming independent of $\epsilon_{p}$ (almost horizontal) and we mark that point as $\epsilon^{*}$. Thus, we find the value $\theta^{*}$ corresponding to the critical point. The values $\epsilon^{*}$ and $\theta^{*}$ are marked with dashed lines in the figure.

\section{APPENDIX G: ISLAND FORMATION AND SANDWICHING NEAR LIPID SATURATION}

We investigate the change in relative free energy as primary islands (PIs) and secondary islands (SIs) merge into island sandwiches (ISs) quantitatively in Fig. 15, considering late-stage $\left(\epsilon_{p} \approx \epsilon_{s} \approx \epsilon_{0}\right)$ lipid adsorption from the outside on a shell with thin top and weak asymmetry. To this end we introduce one more intensive property $\Delta_{s}^{\prime}$ for the case of IS formation:

$$
\Delta_{s}^{\prime}=\frac{G^{\prime}\left(\alpha_{s}=0^{\circ}\right)-G^{\prime}\left(\alpha_{s}=90^{\circ}\right)}{\left|G^{\prime}\left(\alpha_{s}=90^{\circ}\right)\right|},
$$

with $\alpha_{p}=0^{\circ}$. This quantifies the change in $G^{\prime}$ as the secondary side switches from tangential to normal, relative to the original tangential-aligned secondary side, after the primary side has already switched to normal.

A nonoverlapping pair of PIs [Fig. 15(a)] and SIs [Fig. 15(b)] formed around the equator $\left(\theta=90^{\circ}\right)$ has a low 

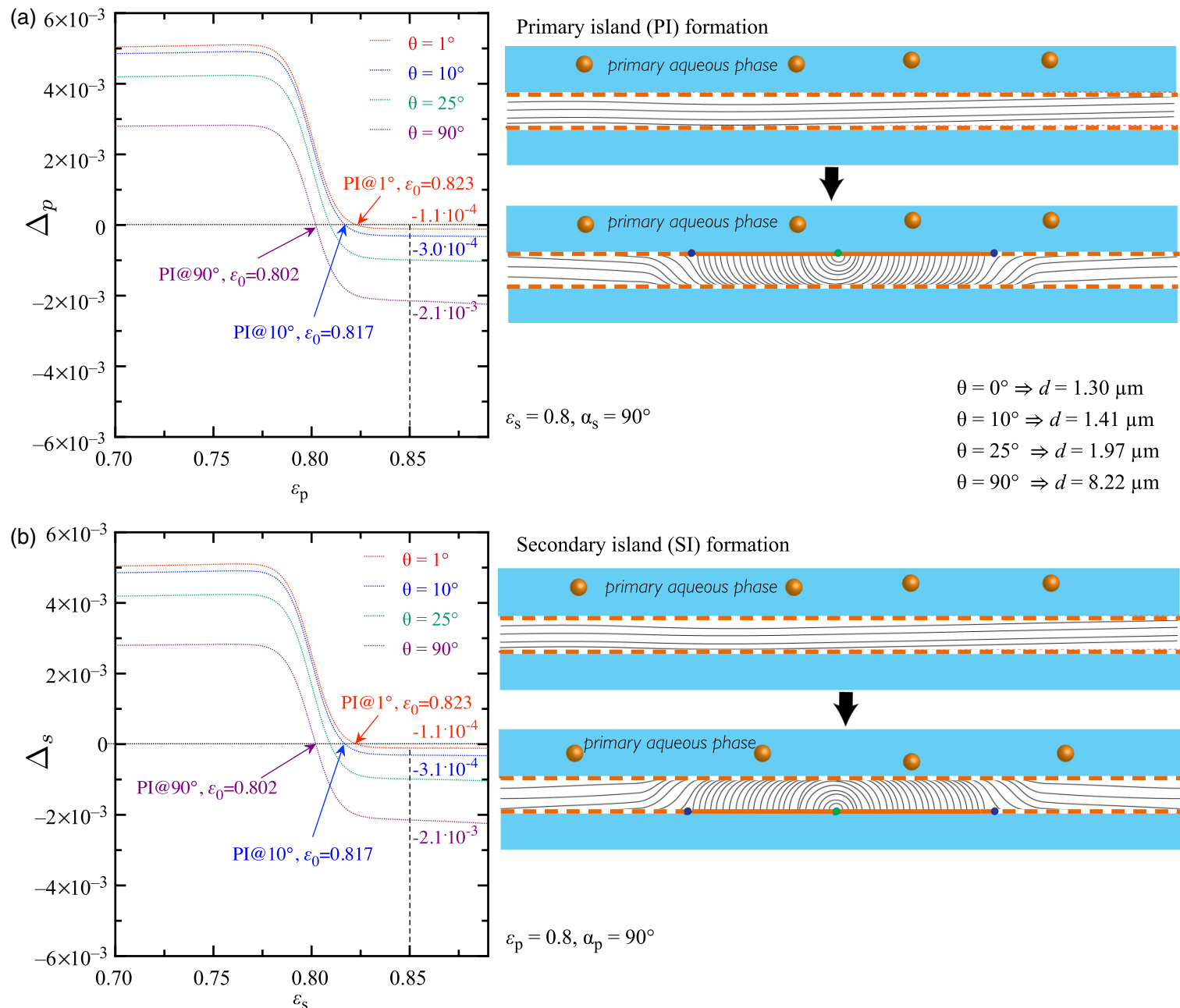

Secondary island (SI) formation

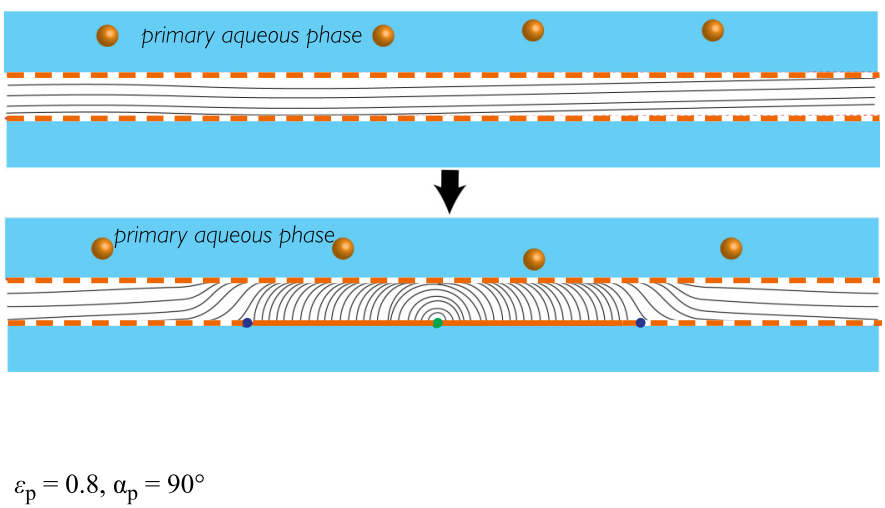

(c)

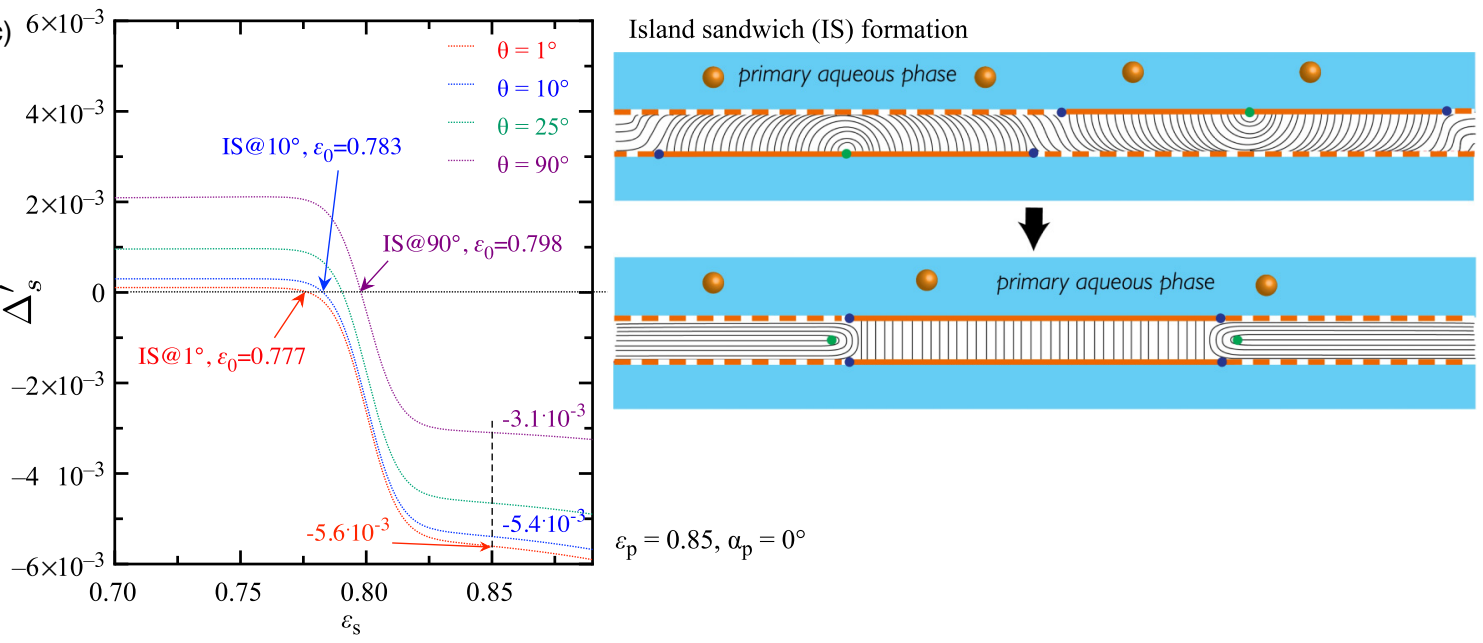

FIG. 15. Relative free energy change for the three types of island formation. The simulations are done for conical cross sections, at polar angle $\theta$, through a weakly asymmetric shell with thin top. Liposomes are considered added to the outer phase, but enough time has passed that both interfaces have reached saturation lipid coverage. Diagram (a) corresponds to primary island (PI) formation with tangential secondary side, $\alpha_{s}=90^{\circ}$, with $\Delta_{p}$ defined in Eq. (3); (b) is the corresponding case for secondary island (SI) formation $\left(\Delta_{p} \rightarrow \Delta_{s}\right)$ with tangential primary side, $\alpha_{p}=90^{\circ}$; (c) corresponds to island sandwich (IS) formation where the primary side is radial $\left(\alpha_{p}=0^{\circ}\right)$, and the secondary side switches from tangential $\left(\alpha_{s}=90^{\circ}\right)$ to radial $\left(\alpha_{s}=0^{\circ}\right), \Delta_{p} \rightarrow \Delta_{s}^{\prime}$. Common parameter values used for all calculations: $R=110 \mu \mathrm{m}, r=102 \mu \mathrm{m}$, $\delta=6.7 \mu \mathrm{m}, \chi=2, A^{*}=0.4 \mathrm{~nm}^{2}, K=10^{-11} \mathrm{~N}, W=20 \mu \mathrm{J} / \mathrm{m}^{2}, B=100, \epsilon_{c}=0.8, T=300 \mathrm{~K}$. 

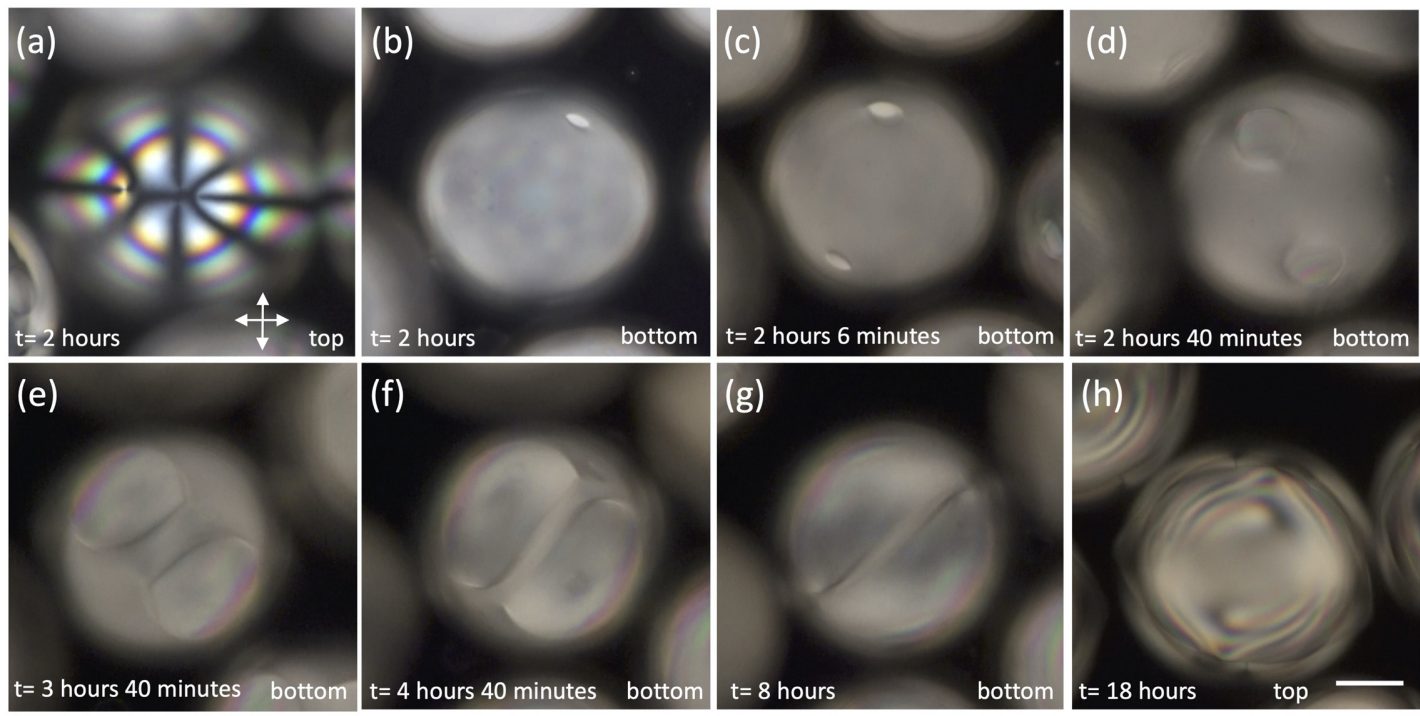

FIG. 16. POM images of a nematic (5CB) shell with $50 \mu \mathrm{M}$ DLPC $(+0.5 \mathrm{wt} \%$ PVA) in the inner phase and 1 wt $\%$ PVA in the outer phase. Image (a) shows the thin side of the shell, with the characteristic collection of topological defects typical of highly asymmetric shells in tangential configuration, $2 \mathrm{hr}$ after production. Around the same time, we observe the first island on the thick side (b), and then another island appears a few minutes later (c). The two islands grow with time $(\mathrm{d}-\mathrm{g})$, and the thick side of the shell changes to hybrid alignment. After 18 $\mathrm{hr}$ we observe that the thin side of the shell also starts changing to hybrid alignment, but the majority remains tangential. Scale bar represents $50 \mu \mathrm{m}$.

average $\bar{\Delta}=\frac{\Delta_{p}\left(\mathrm{PI} @ 90^{\circ}\right)+\Delta_{s}\left(\mathrm{SI} @ 90^{\circ}\right)}{2}=-0.0021$ [calculated at lipid coverages $\epsilon_{p}=0.85$ Fig. 15 (a) and $\epsilon_{s}=0.85$ Fig. 15(b), corresponding to stable islands], as the LC does not violate the normal anchoring imposed by the condensed lipid phase within each island and as the elastic distortion cost at high $d$ is small. The reduction in $\Delta$ by PI-SI overlap into an island sandwich [IS; Fig. 15(c), $\Delta_{s}^{\prime}=-0.0031$ calculated for $\epsilon_{p}=\epsilon_{s}=$ $0.85]$ is only a factor 1.5 , hence the PI-SI attraction is small. However, if the PI and SI are located at $\theta=10^{\circ}$, their average relative energy gain is only $\bar{\Delta}=\frac{\Delta_{p}\left(\mathrm{PI} @ 10^{\circ}\right)+\Delta_{s}\left(\mathrm{SI} @ 10^{\circ}\right)}{2}=$ -0.000305 . While still negative, it is an order of magnitude less prominent. This should be compared with $\Delta=-0.0054$ of the corresponding IS [Fig. 15(c)], indicating a significant energetic drive for bringing the PI and SI into overlap. If we extend this exercise to near the top, at $\theta=1^{\circ}$, we find a 50 -fold relative energy gain by forming an island sandwich.

\section{APPENDIX H: ESTIMATION OF MINIMUM LIPID CONCENTRATION FOR ISLAND FORMATION, WITH PRIMARY INNER PHASE WITH AND WITHOUT PVA}

To estimate the minimum lipid concentration to trigger visible island formation we prepared shells with liposomes mixed into the inner aqueous phase at varying concentrations and studied the texture as a function of time. The minimum concentration yielding island formation this way was $C_{0}=$ $50 \mu \mathrm{M}$; representative snapshots from the slow process are shown in Fig. 16. As established in the main text, in this shell we obtain an apparent maximum possible primary lipid coverage $\epsilon_{p} \approx 0.35<\epsilon_{0}$. Two hours after production, we observe a first island on the thicker side of the shell, and after another minute another island forms on the same side. With time these two islands grow, and after $18 \mathrm{hr}$ the LC alignment on the thicker side has changed to hybrid, an inner boundary still remaining between the islands. The thinner side remains tangential. The $G^{\prime}$ curves for tangential and hybrid alignment in this shell are calculated for $\theta=80^{\circ}$, corresponding to the approximate location of the island birth place in the experiment. The result is shown in Fig. 8.

To confirm that the PVA used for stabilization in most of our experiments gives no significant impact on the lipid adsorption process, we conducted an experiment with inner primary phase twice, on shells produced with and without PVA in the inner phase, respectively. As shown in Fig. 17(a), a shell with $0.1 \mathrm{mM}$ DLPC $+0.5 \mathrm{wt} \%$ PVA in the inner phase shows island formation $50 \mathrm{~min}$ after production, and after $240 \mathrm{~min}$ the thicker side is completely hybrid while the thinner side starts changing alignment from tangential to hybrid. Similarly, we see in Fig. 17(b) that the event sequence and characteristic times are almost the same when the inner phase is without PVA. We conclude that the impact of $0.5 \mathrm{wt} \%$ PVA on the lipid adsorption process is negligible.

\section{APPENDIX I: DERIVATION OF LIPID ADSORPTION DYNAMICS EQUATIONS}

In this Appendix we derive expressions that can be used to compute the coverage fractions of the lipid molecules on the primary $\left(\epsilon_{p}\right)$ as well as on the secondary side $\left(\epsilon_{s}\right)$. To begin, we consider the following dynamics:

$$
C \stackrel{\lambda_{L p}}{\longrightarrow} C_{p} \underset{\lambda_{p s}}{\stackrel{\lambda_{p s}}{\rightleftarrows}} C_{s},
$$

where $C$ is the regular lipid concentration in the liposome solution, while $C_{p}$ and $C_{s}$, respectively, are the corresponding concentrations of lipids on the primary and secondary shell sides. In Eq. (I1) we have assumed that the liposomes break 

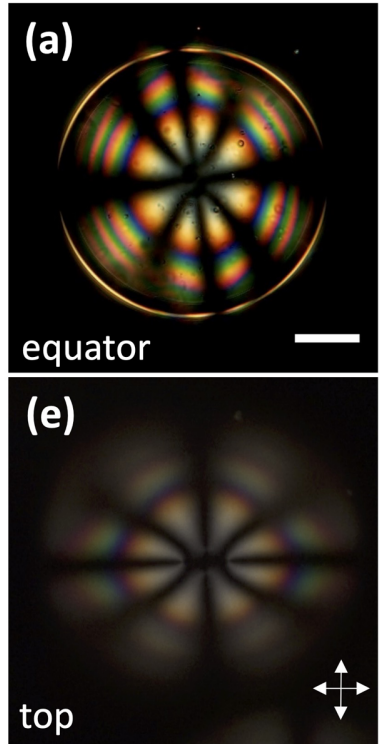
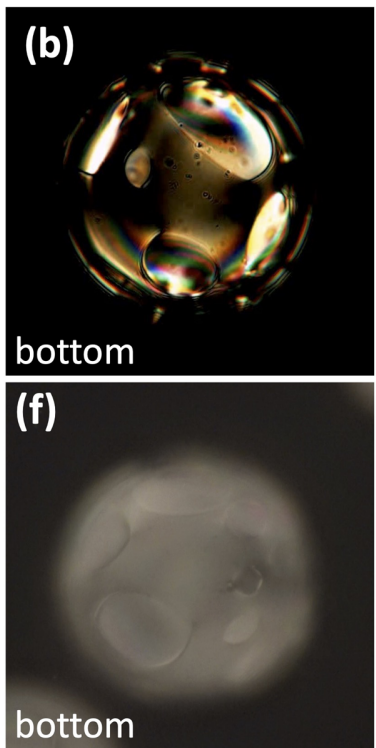

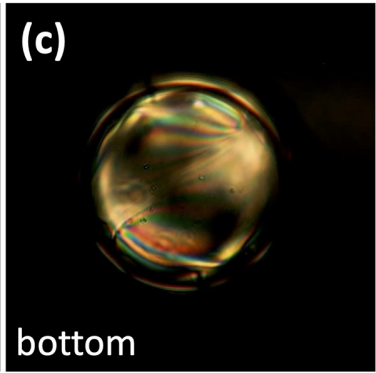

(g)

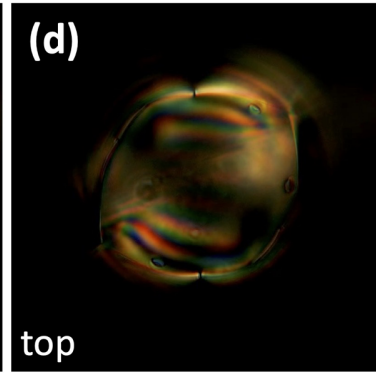

(h)

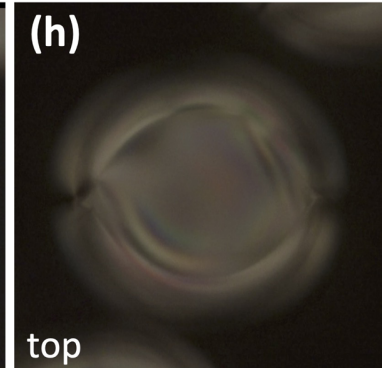

FIG. 17. POM images of 5CB LC shells with $0.1 \mathrm{mM}$ DLPC in the inner phase with (a-d) and without (e-h) 0.5 wt\% PVA at room temperature. After 50 min both shells with and without PVA (a, e) show island growth. Images (b) and (f) show shells after 50 min, and after $80 \mathrm{~min}$, the thick shell side has changed to hybrid alignment (c, g). After around $240 \mathrm{~min}$, alignment on the thinner side also starts changing $(\mathrm{d}, \mathrm{h})$. Scale bars represent $50 \mu \mathrm{m}$. The difference in image quality is due to different microscopes and different cameras being used for observation during the two experiments.

down and produce shell-adsorbed lipid on the primary interface with rate $\lambda_{L p}$, and these lipid molecules can then transfer to the secondary side with rate $\lambda_{p s}$. We further assume that the transfer across the liquid crystal layer is equally likely in both directions, i.e., lipids at the secondary interface can transfer back to the primary interface. For simplicity, we ignore the dependence of the diffusion constant on the director field configuration and we take the same rate for the backward transfer, i.e., $\lambda_{s p}=\lambda_{p s}$ [see Eq. (I1)].

Using Eq. (I1), we can write the rate equations governing the system as follows:

$$
\begin{gathered}
\frac{d C}{d t}=-\lambda_{L p} C, \\
\frac{d C_{p}}{d t}=\lambda_{L p} C+\lambda_{p s}\left(C_{s}-C_{p}\right), \\
\frac{d C_{s}}{d t}=\lambda_{p s}\left(C_{p}-C_{s}\right) .
\end{gathered}
$$

For convenience, we are not writing the time dependence in $C, C_{p}$, and $C_{s}$. The above equations are subject to the initial condition $\left[C(0), C_{p}(0), C_{s}(0)\right]=\left(C_{0}, 0,0\right)$, with $C_{0}$ the DLPC concentration in the primary phase at the start of the experiment.

Solving Eq. (I2) gives

$$
C=C_{0} e^{-\lambda_{L p} t}
$$

To solve for $C_{p}$ and $C_{s}$, we first add Eqs. (I3) and (I4), yielding

$$
\frac{d\left(C_{p}+C_{s}\right)}{d t}=\lambda_{L p} C_{0} e^{-\lambda_{L p} t},
$$

where we have substituted the value of $C$ from Eq. (I5).
Solving Eq. (I6), we get

$$
C_{p}=C_{0}\left(1-e^{-\lambda_{L p} t}\right)-C_{s} .
$$

We substitute the obtained value of $C_{p}$ into Eq. (I4), producing

$$
\frac{d C_{s}}{d t}+2 \lambda_{p s} C_{s}=\lambda_{p s} C_{0}\left(1-e^{-\lambda_{L p} t}\right) .
$$

The solution of the above equation gives the concentration $C_{s}$ of the lipids on the secondary side:

$$
\begin{aligned}
C_{s}= & \frac{C_{0} e^{-t \lambda_{L p}}}{2\left(2 \lambda_{p s}-\lambda_{L p}\right)}\left[2 \lambda_{p s}\left(e^{t \lambda_{L p}}-1\right)\right. \\
& \left.+\lambda_{p s}\left(e^{t\left(\lambda_{L p}-2 \lambda_{p s}\right)}-e^{t \lambda_{L p}}\right)\right] .
\end{aligned}
$$

Substituting $C_{s}$ into Eq. (I7), we obtain

$$
\begin{aligned}
C_{p}= & \frac{C_{0} e^{-t \lambda_{L p}}}{2\left(\lambda_{L p}-2 \lambda_{p s}\right)}\left[\lambda _ { L p } \left(e^{t\left(\lambda_{L p}-2 \lambda_{p s}\right)}\right.\right. \\
& \left.\left.+e^{t \lambda_{L p}}-2\right)-2 \lambda_{p s}\left(e^{t \lambda_{L p}}-1\right)\right] .
\end{aligned}
$$

The fraction $\epsilon_{p}$ is given by

$$
\begin{aligned}
\epsilon_{p} & =\frac{\text { number of lipids at primary interface }}{\text { number of lipids required to cover primary interface }} \\
& =\frac{N_{A} V C_{p}}{A_{\mathrm{LC}} / A^{*}},
\end{aligned}
$$

where $N_{A}$ is the Avogadro's number and $V$ is the volume of solution in contact with the primary interface. While $C_{p}$ refers to adsorbed lipid and is thus not a real solution concentration, it is still, by virtue of the construction through Eq. (I1) and the initial condition, formulated as such, explaining why we multiply it by the external solution volume to obtain the number of moles of adsorbed lipids. 
Since at the time of the first island formation, $t=t_{0}$, we have $\epsilon=\epsilon_{0}$, inverting the above equation gives the relation between the initial lipid concentration and the time at which the first island appears:

$$
C_{0}=2 \epsilon_{0} \frac{A_{\mathrm{LC}}}{N_{A} V A^{*}} \frac{\left(\lambda_{L p}-2 \lambda_{p s}\right) e^{t_{0} \lambda_{L p}}}{\left[\lambda_{L p}\left(e^{t_{0}\left(\lambda_{L p}-2 \lambda_{p s}\right)}+e^{t_{0} \lambda_{L p}}-2\right)-2 \lambda_{p s}\left(e^{t_{0} \lambda_{L p}}-1\right)\right]} .
$$

While expression (I12) should describe the ideal process, the actual experiments are affected by many parameters not included. First, there are other interfaces than shells with which the liposomes may come into contact, e.g., surfaces of vials and pipette tips during manipulation, and also the PVA may interact with lipids in a way that reduces the effective lipid concentration for interaction with the shells. To capture these (and potential other similar) effects leading to loss of effective lipid concentration, we introduce an offset $\Delta C$ in the starting concentration, i.e., $C_{0} \rightarrow C_{0}-\Delta C$.

Moreover, when trying to fit (I12) to the data, we also note that the data appear to follow a behavior as if the experiment started prior to $t=0$. This apparent offset in time can be explained by the unavoidable nonequilibrium conditions that prevail during and immediately after adding the liposome solution, promoting liposome deposition onto shells early in the process. The effect is the same as if the liposomes had had time before we start the experiment to interact with the shells. We capture this artifact by introducing a time offset $\Delta t$, for reasons of compactness incorporated in a rescaled time $\tau=t+\Delta t$ yielding $\tau_{0}=t_{0}+\Delta t$.

Together, these two adjustments lead us to a practical version of (I12) as follows:

$$
\begin{aligned}
C_{0}= & \Delta C+2 \epsilon_{0} \frac{A_{\mathrm{LC}}}{N_{A} V A^{*}} \\
& \times \frac{\left(\lambda_{L p}-2 \lambda_{p s}\right) e^{\tau_{0} \lambda_{L p}}}{\left[\lambda_{L p}\left(e^{\tau_{0}\left(\lambda_{L p}-2 \lambda_{p s}\right)}+e^{\tau_{0} \lambda_{L p}}-2\right)-2 \lambda_{p s}\left(e^{\tau_{0} \lambda_{L p}}-1\right)\right]} .
\end{aligned}
$$

We use (I13) to reproduce the experimental data obtained as described in Appendix J. As also the effective outer phase volume $V$ and the effective shell interface area $A_{\mathrm{LC}}$ cannot be measured very accurately, since density mismatch renders the $8 \mathrm{CB}$ shells floating at the top of the overall sample volume, we have far too many fitting parameters to handle with our scarce data, in particular considering that two of the six data points may correspond to nonequilibrium island formation. We therefore do a manually assisted fitting, where we enter estimates of the effective $A_{\mathrm{LC}}$ and $V$ as well as reasonable values for the decay constants (their inverse value representing the average lifetime of each state), fitting a maximum of four parameters at a time. In this way we find that (I13) can reproduce the experimentally observed behavior well, but we need more data and a more optimized experiment realization to have confidence in the obtained parameter values. These are objectives we are pursuing in our ongoing work.

\section{APPENDIX J: EXPERIMENTAL STUDY OF ISLAND FORMATION DYNAMICS FOR VARYING INITIAL LIPID CONCENTRATION}

We observed shells of $8 \mathrm{CB}$ with different concentrations of lipid, added to the outer phase, varying from 0.007 to $0.25 \mathrm{mM}$. To ensure constant diameter, thickness and number density of shells in all samples, we started with a single batch of shells that was subdivided into six identical samples, each of which was given the same volume of DLPC solution but with different concentrations. These were calculated in order to produce the global $C_{0}$ values indicated on the left in Fig. 18, showing shell textures as a function of time and $C_{0}$. Supplemental Videos 6-11 [21] show the experiments at DLPC concentrations $0.25,0.125,0.06,0.03,0.015$, and $0.007 \mathrm{mM}$, respectively.

At the end of the shell production process, the rapid temperature drop from the isotropic phase to room temperature produces nonequilibrium director field configurations that are transient in 5CB shells, as the nematic phase is fluid enough for the LC to adopt an energyminimizing configuration within seconds to minutes. In $8 \mathrm{CB}$ shells, in contrast, the smectic phase prevailing at room temperature locks in the nonequilibrium configuration, with topological defects trapped in random locations, not just at the thinnest point, where they minimize the free energy.

As mentioned above, we add lipids into the outer phase when the shells are in the smectic phase. Afterwards, the shells are heated to the nematic phase by placing the capillary in a hot stage mounted on the microscope. As shown in the first column of Fig. 18, just after the transition to the nematic phase, trapped defects start moving towards the thinnest shell region to minimize the energy, reaching their destination a few minutes later, as seen in the second column of Fig. 18. We observe one exceptional case at $0.015 \mathrm{mM}$ where one half-integer topological defect remains trapped at the thicker side of the shell.

In all the images we first keep the microscope focus on the thinner side of the shell to monitor the defects as they move towards the energy-minimizing configuration. Once they have settled, we switch the focus to the thicker side where we observe most of the islands forming. From lipid concentrations $0.03-0.25 \mathrm{mM}$ we observe that the rate of islands formation after the first island is quite fast but below $0.03 \mathrm{mM}$ the rate is significantly slower. Thus, we consider the potential impact of kinetically arrested islands forming as a result of fluctuations upon liposome deposition (see Discussion) and therefore tentatively classify these two ranges of concentrations into "equilibrium" $\left(\epsilon_{p}=\epsilon_{0}\right)$ and "nonequilibrium" $\left(\epsilon_{p}<\epsilon_{0}\right)$ island formation, respectively. 


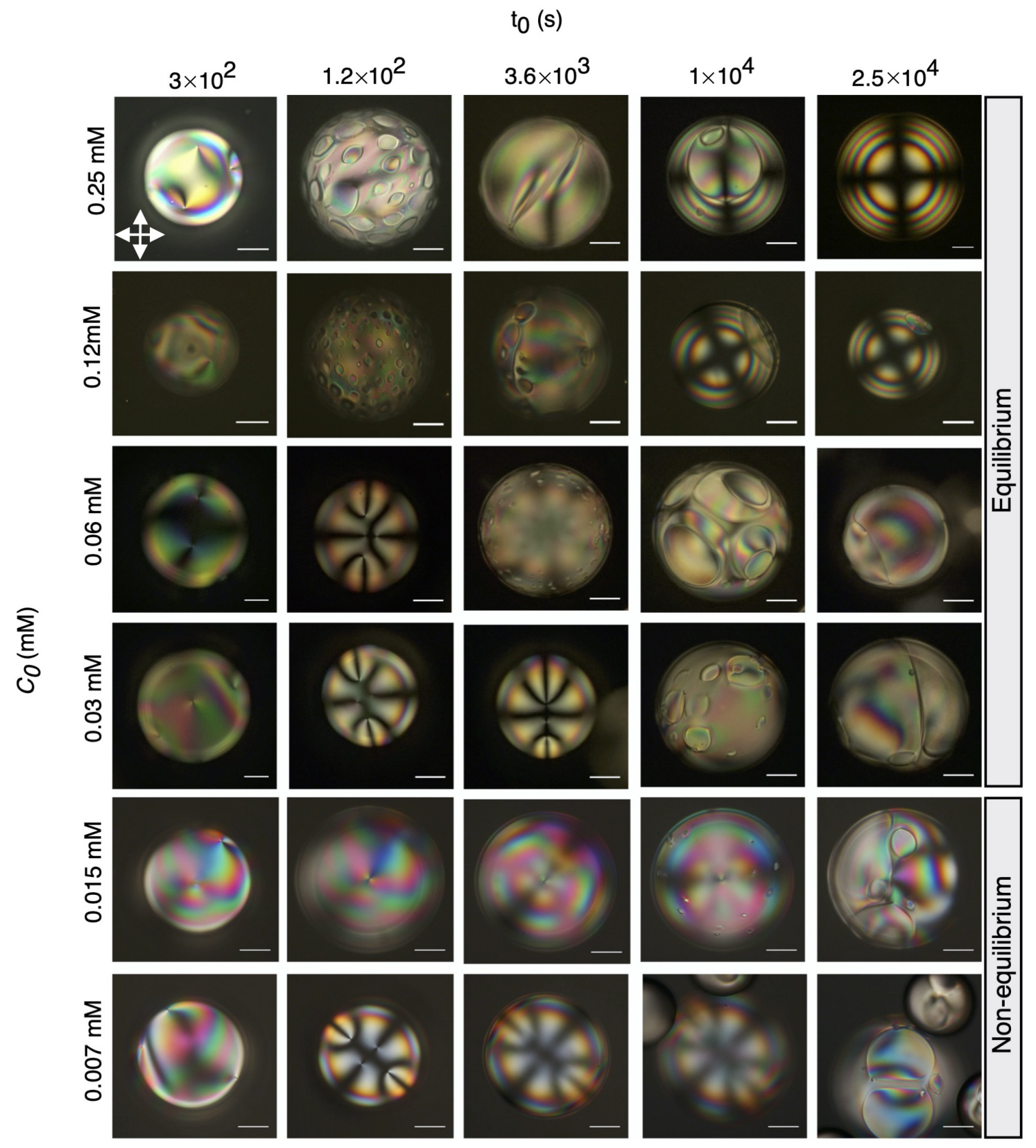

FIG. 18. Island formation vs concentration and time. POM images of $8 \mathrm{CB}$ LC shells in the nematic phase at $34.4^{\circ} \mathrm{C}$ as a function of time $\left(t_{0}\right)$ and DLPC concentration $\left(C_{0}\right)$ in the outer phase. The images show the textural changes as $\mathbf{n}(\mathbf{r})$ changes from fully tangential initially to nonuniform with islands, and eventually uniformly normal if $C_{0}>60 \mu \mathrm{M}$. Scale bars represent $50 \mu \mathrm{m}$.

\section{APPENDIX K: COMPLETE ALIGNMENT CHANGE FROM TANGENTIAL VIA HETEROGENEOUS TO UNIFORMLY NORMAL}

Figure 19 and Supplemental Video 12 [21] show the complete realignment trajectory from tangential to uniformly normal in an 8CB shell exposed to an outer phase DLPC concentration of $0.06 \mathrm{mM}$, the whole process taking about $430 \mathrm{~min}$. The PVA concentration is $1 \mathrm{wt} \%$ in the inner phase and $0.5 \mathrm{wt} \%$ in the outer phase after the liposome solution has been added. In Figs. 19(a) and 19(b) the shell has been cooled to the smectic phase in order to trigger the buckling instability into spherical lunes, revealing the director field throughout the shell. Most other photos are obtained with the shell heated to the nematic phase, the exception being Fig. 19(i), where we again use the instabilities in the smectic phase to confirm the director field configuration. The shell remains island-free [Fig. 19(c)] for more than $20 \mathrm{~min}$, but at around 24 min [Fig. 19(d)] we observe the first island on the thicker shell side. Over the next 2 min many new islands are born on the same side [Fig. 19(e)]. The texture gets increasingly complex [Fig. 19(f)] through the addition of secondary islands once enough lipid has transferred to the inside to ensure $\epsilon_{s}>\epsilon_{0}$. After about $60 \mathrm{~min}$ [Fig. 19(g)], the thick side appears uniformly hybrid-aligned, and after 270 min[Fig. 19(h)] we observe a ring on the thinner side of the shell. To identify the LC alignment within and outside the 

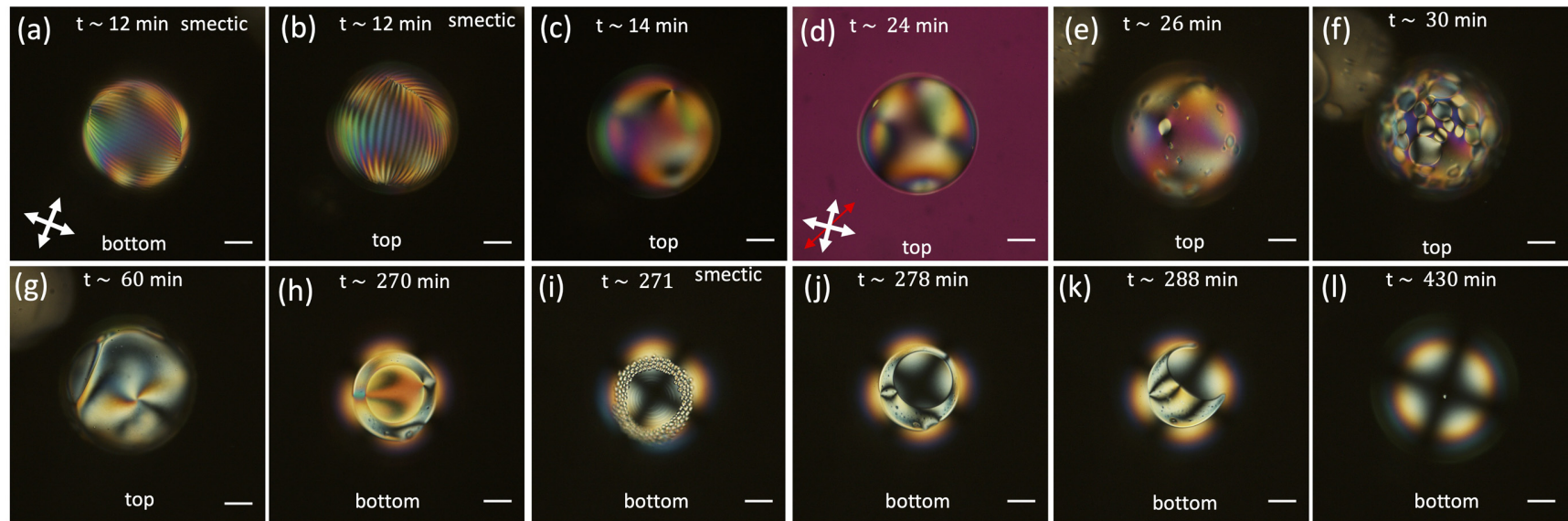

FIG. 19. POM images of an $8 \mathrm{CB}$ shell with $0.06 \mathrm{mM}$ DLPC in the outer phase. The first two images (a, b) show the tangentially aligned $\mathrm{SmA}$ shell at $30^{\circ} \mathrm{C}$ with the focus on the bottom and top, respectively, before the first heating. On heating to $35.4{ }^{\circ} \mathrm{C}$, the shell turns nematic, and in (c) we recognize $+1 / 2$ defects on the shell. Here 24 min after adding DLPC, the shell kept at $35.4{ }^{\circ} \mathrm{C}$, we start seeing islands on the shell (d). Within 2 min the number and size of islands increase (e) and in another 4 min a complex texture with primary and secondary islands is seen (f). Here 60 min after adding DLPC, the thick side is hybrid (g). With time, the thin side (bottom) shows a ring within a shell that is otherwise normal-aligned (h). Cooling the shell to the SmA phase again $\left(30^{\circ} \mathrm{C}\right)$, the characteristic focal conic texture (i) reveals that the LC configuration in the ring is hybrid. We heat the shell back to nematic $\left(35.4^{\circ} \mathrm{C}\right)$ and with time time the hybrid ring shrinks $(\mathrm{j}$, $\mathrm{k})$, leaving the shell fully normal at about $430 \mathrm{~min}$ after the start of the experiment. Scale bars represent $50 \mu \mathrm{m}$.

ring we again cool the shell to the smectic phase, inducing a characteristic focal conic texture within the ring [Fig. 19(i)]. This confirms that the LC is hybrid-aligned here [52], while the shell is normal-aligned everywhere else.

Normal LC alignment in a limited region corresponds to island sandwiches, whereas here this configuration extends throughout most of the shell, showing that the lipid coverage fraction is $\epsilon>\epsilon_{0}$ on both sides except in the ring. Although lipids transfer primarily around the thinnest point, secondary island formation without a corresponding primary island happens mainly at thicker areas to minimize elastic energy. This is the likely early scenario, secondary islands born at

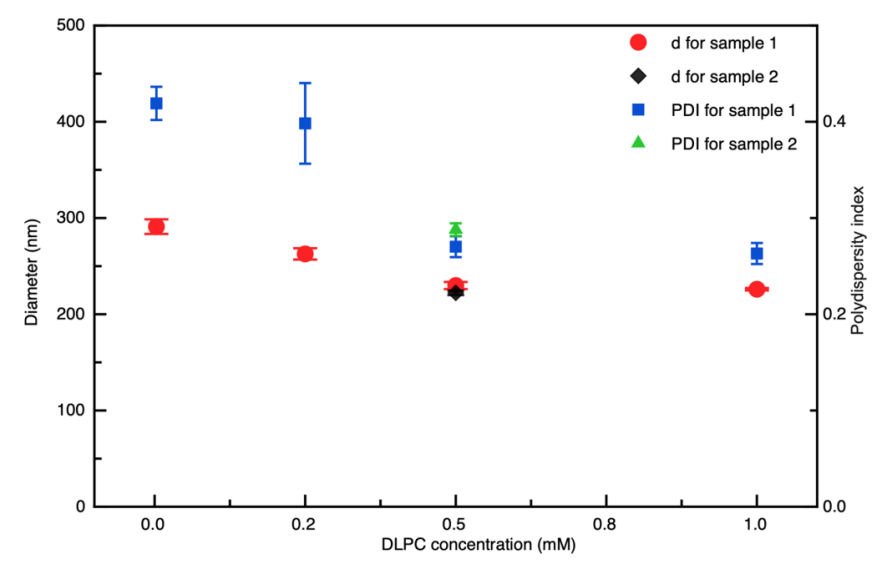

FIG. 20. Liposome diameter $d$ and polydispersity index PDI as measured by DLS, vs DLPC concentration. The left $y$ axis represents the average of liposome diameters. Red dots with error bars show the diameter for the fresh solution (sample 1) and black for the old solution (sample 2). The right $y$ axis represents the polydispersity index (PDI). All blue squares with error bars are the average values of PDI for fresh sample (sample 1) and green triangle for old sample (sample 2). intermediate shell thickness moving slowly towards the thickest point. They may then meet a primary island, forming an island sandwich of minimum elastic energy (the difference is not very large on the thick side; see Fig. 15 and related discussion). Later, when the initially exposed interface is rich with islands, an island sandwich born by direct secondary lipid adsorption at locations already having a primary island is likely, and in that case the energy is reduced rather than increased. The elastic energy contribution thus promotes island sandwich formation, the more so the thinner the shell. The transition to normal alignment on the secondary side is now fastest at the thinnest region of the shell, provided that it exhibits primary islands. We thus have a multistage secondary side realignment sequence where, initially, isolated secondary islands move to the thickest part, whereas later, island sandwiches are born directly onto matching primary islands near the thinnest part. This leaves a final ring of hybrid alignment before the entire shell is normal-aligned. This ring shrinks until the shell is finally fully normally aligned.

\section{APPENDIX L: CHARACTERIZATION OF LIPOSOME SOLUTION BY DYNAMIC LIGHT SCATTERING (DLS)}

Using a Zetasizer Nano S (Malvern Instruments), we performed dynamic light scattering characterization of liposome size on solutions with $0.03,0.25,0.5$, and $1.0 \mathrm{mM}$ DLPC concentration, respectively. The latter was freshly prepared using a tip sonicator (settings as above), and the lower solutions were obtained by dilution of the $1.0 \mathrm{mM}$ stock solution.

For each concentration, we made eight measurements, each of which contained 14 runs, the results summarized in Fig. 20. In the graph, the $x$ axis represents the concentration of DLPC, the left $y$ axis shows the average particle (liposome) diameter (cumulants mean), and the right $y$ axis shows the polydispersity index (PDI) for each measurement. Red circles and black diamonds correspond to the left $y$ axis (diameter), whereas 
blue squares and green triangles relate to the right $y$ axis (PDI). Error bars are the standard deviations calculated from all eight measurements. There is no systematic variation of diameter or PDI with lipid concentration. Averaging over all samples, we obtain a mean diameter of $240 \pm 20 \mathrm{~nm}$. To observe if the size of liposomes changes with time we did DLS measurements for a 2-month-old sample of $0.5 \mathrm{mM}$ DLPC. The black triangle shows the diameter for an old sample and the green triangle shows its PDI. Both values are quite similar to the corresponding values from the fresh sample.
[1] T. Lopez-Leon and A. Fernandez-Nieves, Drops and shells of liquid crystal, Colloid Polym. Sci. 289, 345 (2011).

[2] M. Urbanski, C. G. Reyes, J. Noh, A. Sharma, Y. Geng, V. S. R. Jampani, and J. P. Lagerwall, Liquid crystals in micron-scale droplets, shells and fibers, J. Phys.: Condens. Matter 29, 133003 (2017).

[3] O. Lavrentovich, Topological defects in dispersed liquid crystals, or words and worlds around liquid crystal drops, Liq. Cryst. 24, 117125 (1998).

[4] A. Fernandez-Nieves, V. Vitelli, A. Utada, D. R. Link, M. Marquez, D. R. Nelson, and D. A. Weitz, Novel Defect Structures in Nematic Liquid Crystal Shells, Phys. Rev. Lett. 99, 157801 (2007).

[5] T. Lopez-Leon, V. Koning, K. B. Devaiah, V. Vitelli, and A. Fernandez-Nieves, Frustrated nematic order in spherical geometries, Nat. Phys. 7, 391 (2011).

[6] J. M. Brake, Biomolecular interactions at phospholipiddecorated surfaces of liquid crystals, Science 302, 2094 (2003).

[7] J. Gupta, M. Meli, S. Teren, and N. Abbott, Elastic EnergyDriven Phase Separation of Phospholipid Monolayers at the Nematic Liquid-Crystal-Aqueous Interface, Phys. Rev. Lett. 100, 048301 (2008).

[8] A. D. McNaught and A. Wilkinson, IUPAC. Compendium of Chemical Terminology, 2nd ed. (the "Gold Book") (Blackwell Scientific Publications, Oxford, 1997), https://goldbook.iupac. org/terms/view/P04534.

[9] P. Popov, L. W. Honaker, E. E. Kooijman, E. K. Mann, and A. I. Jákli, A liquid crystal biosensor for specific detection of antigens, Sens. Bio-Sens. Res. 8, 31 (2016).

[10] G. Volovik and O. Lavrentovich, Topological dynamics of defects: Boojums in nematic drops, Sov. Phys. JETP 58, 1159 (1983).

[11] M. C. Carter, D. S. Miller, J. Jennings, X. Wang, M. K. Mahanthappa, N. L. Abbott, and D. M. Lynn, Synthetic mimics of bacterial lipid A trigger optical transitions in liquid crystal microdroplets at ultralow picogram-per-milliliter concentrations, Langmuir 31, 12850 (2015).

[12] P. Bao, D. A. Paterson, P. L. Harrison, K. Miller, S. Peyman, J. C. Jones, J. Sandoe, S. D. Evans, R. J. Bushby, and H. F. Gleeson, Lipid coated liquid crystal droplets for the on-chip detection of antimicrobial peptides, Lab Chip 19, 1082 (2019).

[13] M. Zhang and C. H. Jang, Sensitive detection of trypsin using liquid-crystal droplet patterns modulated by interactions between poly-L-lysine and a phospholipid monolayer, ChemPhysChem 15, 2569 (2014).

[14] A. Sharma, A. M. Stoffel, and J. P. F. Lagerwall, Liquid crystal elastomer shells with topological defect-defined actuation: Complex shape morphing, opening/closing, and unidirectional rotation, J. Appl. Phys. 129, 174701 (2021).

[15] V. S. R. Jampani, R. H. Volpe, K. Reguengo de Sousa, J. Ferreira Machado, C. M. Yakacki, and J. P. F. Lagerwall, Liquid crystal elastomer shell actuators with negative order parameter, Sci. Adv. 5, eaaw2476 (2019).

[16] V. S. R. Jampani, D. J. Mulder, K. R. De Sousa, A.-H. Glbart, J. P. F. Lagerwall, and A. P. H. J. Schenning, Micrometerscale porous buckling shell actuators based on liquid crystal networks, Adv. Funct. Mater. 28, 1801209 (2018).

[17] H. Fuster, X. Wang, X. Wang, E. Bukusoglu, S. Spagnolie, and N. Abbott, Programming van der Waals interactions with complex symmetries into microparticles using liquid crystallinity, Sci. Adv. 6, eabb1327 (2020).

[18] S. Martin and R. G. Parton, Lipid droplets: A unified view of a dynamic organelle, Nat. Rev. Mol. Cell Biol. 7, 373 (2006).

[19] C. Kataoka-Hamai and K. Kawakami, Interaction mechanisms of giant unilamellar vesicles with hydrophobic glass surfaces and silicone oil-water interfaces: Adsorption, deformation, rupture, dynamic shape changes, internal vesicle formation, and desorption, Langmuir 35, 16136 (2019).

[20] M. Tsuei, M. Shivrayan, Y. Kim, S. Thayumanavan, and N. Abbott, Optical blinking triggered by collisions of single supramolecular assemblies of amphiphilic molecules with interfaces of liquid crystals, J. Am. Chem. Soc. 142, 61396148 (2020).

[21] See Supplemental Material at http://link.aps.org/supplemental/ 10.1103/PhysRevResearch.4.013130 for videos showing the full dynamic response of LC shells to lipid exposure under varying conditions, corresponding to the discussion in the paper where the movies are referenced; further description is provided in the Read Me file.

[22] T. Baumgart, S. Hess, and W. Webb, Imaging coexisting fluid domains in biomembrane models coupling curvature and line tension, Nature (London) 425, 821 (2003).

[23] S. T. Lagerwall, On some important chapters in the history of liquid crystals, Liq. Cryst. 40, 1698 (2013).

[24] T. J. Sluckin, D. A. Dunmur, and H. Stegemeyer, Crystals That Flow: Classic Papers from the History of Liquid Crystals (Taylor and Francis, London, 2004).

[25] L. Tran, M. O. Lavrentovich, G. Durey, A. Darmon, M. F. Haase, N. Li, D. Lee, K. J. Stebe, R. D. Kamien, and T. LopezLeon, Change in Stripes for Cholesteric Shells via Anchoring in Moderation, Phys. Rev. X 7, 041029 (2017).

[26] M. Schadt and F. Muller, Physical properties of new liquidcrystal mixtures and electrooptical performance in twisted nematic displays, IEEE Trans. Electron Devices 25, 1125 (1978).

[27] G.-P. Chen, H. Takezoe, and A. Fukuda, Determination of $K_{i}$ ( $i=1-3)$ and $\mu_{j}(j=2-6)$ in 5CB by observing the angular dependence of Rayleigh line spectral widths, Liq. Cryst. 5, 341 (1989).

[28] J. Noh, Y. Wang, H.-L. Liang, V. S. R. Jampani, A. Majumdar, and J. P. F. Lagerwall, Dynamic tuning of the director field 
in liquid crystal shells using block copolymers, Phys. Rev. Research 2, 033160 (2020).

[29] A. Sonin, Inorganic lyotropic liquid crystals, J. Mater. Chem. 8, 2557 (1998).

[30] P. Prinsen and P. van der Schoot, Shape and director-field transformation of tactoids, Phys. Rev. E 68, 021701 (2003).

[31] Y. Kim, S. Shiyanovskii, and O. Lavrentovich, Morphogenesis of defects and tactoids during isotropic-nematic phase transition in self-assembled lyotropic chromonic liquid crystals, J. Phys.: Condens. Matter 25, 404202 (2013).

[32] A. Mangiarotti, B. Caruso, and N. Wilke, Phase coexistence in films composed of DLPC and DPPC: A comparison between different model membrane systems, Biochim. Biophys. Acta 1838, 1823 (2014).

[33] A. Sharma and J. P. Lagerwall, Influence of head group and chain length of surfactants used for stabilising liquid crystal shells, Liq. Cryst. 45, 2319 (2018).

[34] A. Sharma, V. S. R. Jampani, and J. P. F. Lagerwall, Realignment of liquid crystal shells driven by temperature-dependent surfactant solubility, Langmuir 35, 11132 (2019).

[35] J. N. Israelachvili, Intermolecular and Surface Forces, 3rd ed. (Academic Press, Burlington, MA, 2010).

[36] J. Cumberland, T. Lopatkina, M. Murachver, P. Popov, V. Kenderesi, Á. Buka, E. Mann, and A. Jákli, Bending nematic liquid crystal membranes with phospholipids, Soft Matter 14, 7003 (2018).

[37] R. Meister, H. Dumoulin, M.-A. Hallé, and P. Pieranski, The anchoring of a cholesteric liquid crystal at the free surface, J. Phys. II (France) 6, 827 (1996).

[38] I. B. Liu, M. A. Gharbi, V. L. Ngo, R. D. Kamien, S. Yang, and K. J. Stebe, Elastocapillary interactions on nematic films, Proc. Natl. Acad. Sci. U. S. A. 112, 6336 (2015).

[39] M. O. Lavrentovich and L. Tran, Undulation instabilities in cholesteric liquid crystals induced by anchoring transitions, Phys. Rev. Research 2, 023128 (2020).

[40] P. Poulin, H. Stark, T. C. Lubensky, and D. A. Weitz, Novel colloidal interactions in anisotropic fluids, Science 275, 1770 (1997).
[41] I. Musevic, M. Skarabot, U. Tkalec, M. Ravnik, and S. Zumer, Two-dimensional nematic colloidal crystals self-assembled by topological defects, Science 313, 954 (2006).

[42] D. Myung and S. Park, Optical properties and applications of photonic shells, ACS Appl. Mater. Interfaces 11, 2035020359 (2019).

[43] K. He, F. Campo-Cortés, M. Goral, T. López-León, and J. M. Gordillo, Micron-sized double emulsions and nematic shells generated via tip streaming, Phys. Rev. Fluids 4, 124201 (2019).

[44] J. K. Gupta and N. L. Abbott, Principles for manipulation of the lateral organization of aqueous-soluble surface-active molecules at the liquid crystal-aqueous interface, Langmuir 25, 2026 (2009).

[45] M. P. Murrell, R. Voituriez, J.-F. Joanny, P. Nassoy, C. Sykes, and M. L. Gardel, Liposome adhesion generates traction stress, Nat. Phys. 10, 163 (2014).

[46] A. S. Utada, E. Lorenceau, D. R. Link, P. D. Kaplan, H. A. Stone, and D. A. Weitz, Monodisperse double emulsions generated from a microcapillary device, Science 308, 537 (2005).

[47] P. Bao, M. R. Cheetham, J. S. Roth, A. C. Blakeston, R. J. Bushby, and S. D. Evans, On-chip alternating current electrophoresis in supported lipid bilayer membranes, Anal. Chem. 84, 10702 (2012).

[48] https://doi.org/10.5281/zenodo.5893857.

[49] H. L. Liang, S. Schymura, P. Rudquist, and J. Lagerwall, Nematic-Smectic Transition under Confinement in Liquid Crystalline Colloidal Shells, Phys. Rev. Lett. 106, 247801 (2011).

[50] T. Lopez-Leon, A. Fernandez-Nieves, M. Nobili, and C. Blanc, Nematic-Smectic Transition in Spherical Shells, Phys. Rev. Lett. 106, 247802 (2011).

[51] M. Rubinstein and R. H. Colby, Polymer Physics (Chemistry) (Oxford University Press, Oxford, 2003), p. 454.

[52] H. L. Liang, R. Zentel, P. Rudquist, and J. Lagerwall, Towards tunable defect arrangements in smectic liquid crystal shells utilizing the nematic-smectic transition in hybrid-aligned geometries, Soft Matter 8, 5443 (2012). 